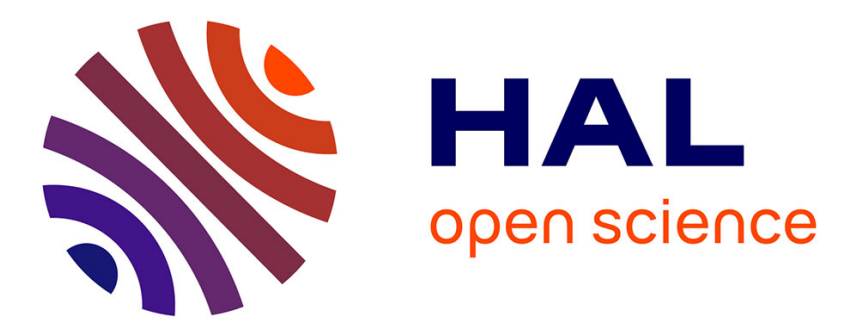

\title{
Space Shift Keying (SSK-) MIMO over Correlated Rician Fading Channels: Performance Analysis and a New Method for Transmit-Diversity
}

Marco Di Renzo, Harald Haas

\section{- To cite this version:}

Marco Di Renzo, Harald Haas. Space Shift Keying (SSK-) MIMO over Correlated Rician Fading Channels: Performance Analysis and a New Method for Transmit-Diversity. IEEE Transactions on Communications, 2011, 59 (1), pp.116-129. 10.1109/TCOMM.2011.111710.090775 . hal-00658681

\section{HAL Id: hal-00658681 \\ https://hal-centralesupelec.archives-ouvertes.fr/hal-00658681}

Submitted on 20 Jan 2012

HAL is a multi-disciplinary open access archive for the deposit and dissemination of scientific research documents, whether they are published or not. The documents may come from teaching and research institutions in France or abroad, or from public or private research centers.
L'archive ouverte pluridisciplinaire HAL, est destinée au dépôt et à la diffusion de documents scientifiques de niveau recherche, publiés ou non, émanant des établissements d'enseignement et de recherche français ou étrangers, des laboratoires publics ou privés. 


\title{
Space Shift Keying (SSK-) MIMO over Correlated Rician Fading Channels: Performance Analysis and a New Method for Transmit-Diversity
}

\author{
Marco Di Renzo, Member, IEEE, and Harald Haas, Member, IEEE
}

\begin{abstract}
In this paper, we study the performance of Space Shift Keying (SSK) modulation for a generic Multiple-InputMultiple-Output (MIMO) wireless system over correlated Rician fading channels. In particular, our contribution is twofold. i) First, we propose a very general framework for computing the Average Bit Error Probability (ABEP) of SSK-MIMO systems over a generic Rician fading channel with arbitrary correlation and channel parameters. The framework relies upon the Moschopoulos method. We show that it is exact for MIMO systems with two transmit-antenna and arbitrary receive-antenna, while an asymptotically-tight upper-bound is proposed to handle the system setup with an arbitrary number of transmit-antenna. ii) Second, moving from the consideration that conventional SSKMIMO schemes can offer only receive-diversity gains, we propose a novel SSK-MIMO scheme that can exploit the transmitantenna to increase the diversity order. The new method has its basic foundation on the transmission of signals with good time-correlation properties, and is called Time-OrthogonalSignal-Design (TOSD-) assisted SSK modulation (TOSD-SSK). It is shown that the proposed method can increase twofold the diversity order for arbitrary transmit- and receive-antenna. In particular, for MIMO systems with two transmit-antenna and $N_{r}$ receive-antenna full-diversity equal to $2 N_{r}$ can be achieved. Analytical frameworks and theoretical findings are substantiated via Monte Carlo simulations for various system setups.
\end{abstract}

Index Terms-Spatial Modulation (SM), Space Shift Keying (SSK) modulation, Multiple-Input-Multiple-Output (MIMO) systems, transmit-diversity, performance analysis, correlated Rician fading channels.

\section{INTRODUCTION}

$\mathbf{S}$ PATIAL Modulation (SM) has been recently proposed as a new and promising candidate transmission technique for low-complexity implementations of Multiple-Input-MultipleOutput (MIMO) wireless systems that require a medium/high transmission rate [1], [2]. In particular, it has been shown that $\mathrm{SM}$ is an excellent solution to solve the three main issues

Paper approved by A. Zanella, the Editor for Wireless Systems of the IEEE Communications Society. Manuscript received December 17, 2009; revised May 12, 2010; accepted August 18, 2010.

This paper was presented in part at the ITG/IEEE International Workshop on Smart Antennas (WSA), Bremen, Germany, February 2010, and at the IEEE International Conference on Communications (ICC), Cape Town, South Africa, May 2010.

M. Di Renzo is with L2S, UMR 8506 CNRS - SUPELEC - Univ ParisSud, 3 rue Joliot-Curie, 91192 Gif-sur-Yvette CEDEX (Paris), France (email: marco.direnzo@1ss.supelec.fr).

H. Haas is with The University of Edinburgh, College of Science and Engineering, School of Engineering, Institute for Digital Communications (IDCOM), Alexander Graham Bell Building, King's Buildings, Mayfield Road, Edinburgh, EH9 3JL, Scotland, United Kingdom (UK) (e-mail: h.haas@ed.ac.uk)

Digital Object Identifier XX.XXXX/TCOMM.XXXX.XX.XXXXXX. amongst the adoption of MIMO systems for low-complexity and power-efficient applications, i.e., i) inter-channel interference, ii) inter-antenna synchronization, and iii) multiple radio frequency chains at the transmitter [3]-[6]. Numerical results in [3], [4], [6] have shown that SM can offer better performance and a reduced computational complexity than other popular MIMO schemes for fading channels. Furthermore, in [6] a low-complexity implementation of SM, which is called Space Shift Keying (SSK) modulation, has been suggested and studied. SSK modulation offers a good solution to trade-off receiver complexity for data rate.

The working principle of SM for MIMO systems is based on three main fundamental assumptions: i) the wireless environment naturally modulates the transmitted signal, ii) each transmit-receive wireless link has a different channel, and iii) the receiver employs the a priori channel knowledge to detect the transmitted signal. In other words, SM takes advantage of the location-specific property of the wireless channel, i.e., the uniqueness of each transmit-receive wireless link, for communication [1], [7]. This property is exploited by adopting a simple but effective coding mechanism that establishes a one-to-one mapping between blocks of information bits to be transmitted and the spatial positions of the transmit-antenna in the antenna-array [4]. More specifically, the basic idea of SM is to map a block of information bits into two information carrying units: 1) a symbol that is chosen from a complex signalconstellation diagram, and 2) a unique transmit-antenna index that is chosen from the set of transmit-antenna in the antennaarray. SM allows, at any time instance, just one transmitantenna to be switched on for data transmission and keeps all the other transmit-antenna silent. Thus, SM entirely avoids inter-channel interference, requires no synchronization among the transmit-antenna, and needs only one radio frequency chain for data transmission: these features make it wellsuited for low-complexity MIMO implementations. Moreover, conveying part of the information in the transmit-antenna index introduces a spatial multiplexing gain with respect to single-antenna systems [4].

Owing to its peculiar working principle, SM is a very new transmission technique for data communication over wireless channels. However, research in this field is still at its infancy and fundamental issues still need to be addressed to assess the possibilities of exploitation of this technology over realistic propagation environments and for practical operating conditions. In particular, in the current technical literature there is a lack of sound communication-theoretic 
frameworks for the analysis, design, and optimization of this novel transmission technology. Some notable results available to date are as follows: i) in [4], the authors have proposed an approximated semi-analytical framework to compute the Average Bit Error Probability (ABEP) of an ad hoc receiver design over independent and identically distributed (i.i.d.) Rayleigh fading channels; ii) the latter framework has been extended to correlated Nakagami- $m$ fading channels in [8]. However, fading correlation is taken into account only for data detection, while the probability of transmit-antenna detection is computed by using the framework in [4], which neglects fading correlation; iii) in [5], [6] the ABEP of the MaximumLikelihood (ML) detector for SM and SSK modulation, respectively, has been computed for i.i.d. Rayleigh fading channels; iv) the framework in [6] has been generalized, for the same channel model, in [9] by assuming that more than one antenna can be switched on for data transmission; and v) in [10], [11], we have recently computed the ABEP of SSK modulation over correlated and non-identically distributed Nakagami- $m$ fading channels for optimal and sub-optimal detectors, respectively. However, these latter frameworks are limited to MIMO systems with just one receive-antenna. The latest developments about analysis and design of SM and SSK modulation over fading channels are available in [12]-[16].

In the depicted context, the novel contribution of this paper is twofold.

1) We propose a general analytical framework to compute the ABEP of SSK-MIMO systems over Rician fading channels with arbitrary channel correlation and fading parameters. The ML-optimum detector in [6] is considered. It is shown that the framework is useful for MIMO systems with arbitrary transmit- and receive-antenna. In particular, by exploiting the Moschopoulos method [17], it is shown that an exact closed-form expression of the ABEP can be derived when the transmitter is equipped with two antennas, while a tight upper-bound can be obtained for arbitrary transmit-antenna. This framework overcomes all the restrictions of previously proposed frameworks on either the channel model [4][8] or the number of antennas at the receiver [10], [11]. In particular, channel correlation and power imbalance are taken into account.

2) Looking into the various forms of SM and SSK modulation proposed so far, it can be readily argued that all of them provide only receive-diversity gains, while the transmit-antenna are used for enabling data transmission only. A sound proof of this result can be found in [6] for i.i.d. Rayleigh fading. However, it is well-known that several methods to achieve transmit-diversity gains exist for MIMO schemes, e.g., [18], [19], and [20, Sec. III] for a survey. Motivated by this consideration, we propose a novel SSK modulation scheme, called TimeOrthogonal-Signal-Design (TOSD-) assisted SSK modulation (TOSD-SSK), which can offer transmit-diversity gains. The basic idea behind the TOSD-SSK modulation scheme is to optimize the shape of the signals emitted by the transmit-antenna to satisfy some orthogonality constrains in the time domain. We will show that the proposed method can increase twofold the diversity order of conventional SSK-MIMO systems with the same number of transmit- and receive-antenna. In particular, for MIMO systems with two transmit-antenna and $N_{r}$ receive-antenna full-diversity equal to $2 N_{r}$ can be achieved. A framework to compute the ABEP of this proposed scheme is also given. Furthermore, with respect to the many techniques proposed for transmitdiversity purposes so far [20, Sec. III], our method retains the main distinguishable feature of SM: the multiple-antenna at the transmitter are not required to transmit simultaneously, but just one of them is active at any time instance while all the other antennas are kept silent. This way, the TOSD-SSK modulation scheme requires neither inter-antenna synchronization nor multiple radio frequency chains at the transmitter: transmitdiversity gains are achieved since the information is encoded into the spatial position of the transmit-antenna and not because redundant replicas of the same message are transmitted.

The remainder of this paper is organized as follows. In Section II, system and channel models are introduced. In Section III, the analytical framework for performance analysis of SSK-MIMO systems over correlated Rician fading channels is developed. In Section IV, the new TOSD-SSK-MIMO scheme with transmit-diversity gains is introduced and its performance is analyzed over generically correlated Rician fading channels. In Section V, numerical and simulation results are shown to substantiate the accuracy of the analytical frameworks and the increased diversity order offered by the new SSK modulation scheme. Finally, Section VI concludes the paper.

\section{SySTEM MOdEL}

Let us consider a generic $N_{t} \times N_{r}$ MIMO system, with $N_{t}$ and $N_{r}$ being the number of transmit- and receive-antenna, respectively. SSK modulation works as follows [4], [6]: i) the transmitter encodes blocks of $\log _{2}\left(N_{t}\right)$ data bits into the index of a single transmit-antenna (i.e., antenna-index coded modulation), which is switched on for data transmission while all the other antennas are kept silent, and ii) the receiver solves a $N_{t}$-hypothesis detection problem to estimate the transmitantenna that is not idle, which results in the estimation of the unique sequence of bits emitted by the encoder. Throughout this paper, the unique block of bits encoded into the index of the $i$-th transmit-antenna is called "message" and is denoted by $m_{i}$ for $i=1,2, \ldots, N_{t}$. The $N_{t}$ messages are assumed to be emitted with equal probability by the encoder. Moreover, the related transmitted signal is denoted by $s_{i}(\cdot)$ for $i=$ $1,2, \ldots, N_{t}$. It is implicitly assumed in this notation that, if $m_{i}$ is transmitted, the analog signal $s_{i}(\cdot)$ is emitted by the $i$-th transmit-antenna while all the other transmit-antenna radiate no power.

\section{A. Notation}

Let us briefly introduce the main notation used in what follows. i) We adopt a complex-envelope signal representation. ii) $j=\sqrt{-1}$ is the imaginary unit. iii) $(\cdot)^{*}$ and 
$(\cdot)^{T}$ denote complex-conjugate and transpose operators, respectively. iv) $(x \otimes y)(t)=\int_{-\infty}^{+\infty} x(\xi) y(t-\xi) d \xi$ is the convolution of signals $x(\cdot)$ and $y(\cdot) . \mathrm{v})|\cdot|^{2}$ denotes square absolute value. vi) $\mathrm{E}\{\cdot\}$ is the expectation operator. vii) $\operatorname{Re}\{\cdot\}$ and $\operatorname{Im}\{\cdot\}$ denote the real and imaginary part operators, respectively. viii) $\operatorname{Pr}\{\cdot\}$ denotes probability. ix) $Q(x)=$ $(1 / \sqrt{2 \pi}) \int_{x}^{+\infty} \exp \left(-t^{2} / 2\right) d t$ is the Q-function. x) $\hat{m}$ denotes the message estimated at the receiver-side. xi) $E_{m}$ is the average energy transmitted by each antenna that emits a non-zero signal. xii) $T_{m}$ denotes the signaling interval for each information message $m_{i}$ for $i=1,2, \ldots, N_{t}$. xiii) The noise $n_{l}$ at the input of the $l$-th receive-antenna $\left(l=1,2, \ldots, N_{r}\right)$ is assumed to be an Additive White Gaussian Noise (AWGN) process, with both real and imaginary parts having a power spectral density equal to $N_{0}$. Across the receive-antenna, the noises $n_{l}$ for $l=1,2, \ldots, N_{r}$ are statistically independent. xiv) For ease of notation, we set $\bar{\gamma}=E_{m} /\left(4 N_{0}\right)$. xv) $X \sim$ $N\left(\mu, \sigma^{2}\right)$ denotes a Gaussian Random Variable (RV) with mean $\mu$ and standard deviation $\sigma$. xvi) Matrices and vectors are denoted in boldface. xvii) $\Gamma(\cdot)$ is the Gamma function [21, Eq. (6.1.1)]. xviii) $\delta(\cdot)$ and $\delta_{.,}$, are the Dirac and Kronecker delta functions, respectively. xix) $G_{p, q}^{m, n}\left(. \mid\left(\begin{array}{l}\left(a_{p}\right) \\ \left(b_{q}\right)\end{array}\right)\right.$ is the Meijer-G function defined in [22, Ch. 8, pp. 519]. xx) $f_{X}(\cdot)$ and $M_{X}(s)=\mathrm{E}\{\exp (-s X)\}$ denote Probability Density Function (PDF) and Moment Generating Function (MGF) of RV X, respectively.

\section{B. Channel Model}

We consider a frequency-flat slowly-varying channel with generically correlated and non-identically distributed Rician fading over the transmit-receive wireless links. In particular:

- $h_{i, l}(t)=\alpha_{i, l} \delta\left(t-\tau_{i, l}\right)$ is the channel impulse response of the transmit-receive wireless link from the $i$-th transmit-antenna to the $l$-th receive-antenna for $i=1,2, \ldots, N_{t}$ and $l=1,2, \ldots, N_{r} . \alpha_{i, l}$ and $\tau_{i, l}$ are the complex channel gains and time-delays, respectively.

- The delays $\tau_{i, l}$ for $i=1,2, \ldots, N_{t}$ and $l=1,2, \ldots, N_{r}$ are assumed to be i.i.d. in $\left[0, T_{m}\right)$, but known at the receiver, i.e., perfect time-synchronization is considered.

- The complex channel gains $\alpha_{i, l}$ for $i=1,2, \ldots, N_{t}$ and $l=1,2, \ldots, N_{r}$ are defined as $\alpha_{i, l}=\beta_{i, l} \exp \left(j \varphi_{i, l}\right)=$ $\alpha_{i, l}^{R}+j \alpha_{i, l}^{I}$, where $\alpha_{i, l}^{R}=\operatorname{Re}\left\{\alpha_{i, l}\right\}, \alpha_{i, l}^{I}=\operatorname{Im}\left\{\alpha_{i, l}\right\}$, $\beta_{i, l}=\sqrt{\left(\alpha_{i, l}^{R}\right)^{2}+\left(\alpha_{i, l}^{I}\right)^{2}}$ is the fading envelope, and $\varphi_{i, l}=\arctan \left(\alpha_{i, l}^{I} / \alpha_{i, l}^{R}\right)$ is the channel phase.

- By assuming a Rician fading channel model [23], we have $\alpha_{i, l}^{R} \sim N\left(\mu_{i, l}, \sigma_{i, l}^{2}\right)$ and $\alpha_{i, l}^{I} \sim N\left(\mu_{i, l}, \sigma_{i, l}^{2}\right)$ for $i=1,2, \ldots, N_{t}$ and $l=1,2, \ldots, N_{r}$. Accordingly, $K_{R}^{(i, l)}=\mu_{i, l}^{2} /\left(2 \sigma_{i, l}^{2}\right)$ and $\Omega_{i, l}=\mu_{i, l}^{2}+2 \sigma_{i, l}^{2}$ are the Rice factor and the normalized ${ }^{1}$ average Signal-to-NoiseRatio (SNR) of the transmit-receive wireless link from the $i$-th transmit-antenna to the $l$-th receive-antenna for $i=1,2, \ldots, N_{t}$ and $l=1,2, \ldots, N_{r}$, respectively.

\footnotetext{
${ }^{1} \Omega_{i, l}$ is normalized to the transmit-energy $\left(E_{m}\right)$ and the noise power $\left(N_{0}\right)$ at the receiver input.
}

In particular, $K_{R}^{(i, l)}$ and $\Omega_{i, l}$ for $i=1,2, \ldots, N_{t}$ and $l=1,2, \ldots, N_{r}$ might not be the same over different transmit-receive wireless links.

- A generic channel correlation model is assumed. In particular, the correlation coefficient between the transmitreceive wireless link from the $i_{1}$-th transmit-antenna to the $l_{1}$-th receive-antenna for $i_{1}=1,2, \ldots, N_{t}$ and $l_{1}=1,2, \ldots, N_{r}$, and the transmit-receive wireless link from the $i_{2}$-th transmit-antenna to the $l_{2}$-th receiveantenna for $i_{2}=1,2, \ldots, N_{t}$ and $l_{2}=1,2, \ldots, N_{r}$ is:

$$
\rho_{\left(i_{1}, i_{2}\right)}^{A B}\left(l_{1}, l_{2}\right)=\frac{\mathrm{E}\left\{\left(\alpha_{i_{1}, l_{1}}^{A}-\mu_{i_{1}, l_{1}}\right)\left(\alpha_{i_{2}, l_{2}}^{B}-\mu_{i_{2}, l_{2}}\right)^{*}\right\}}{\sigma_{i_{1}, l_{1}} \sigma_{i_{2}, l_{2}}}
$$

where $A=\{R, I\}$ and $B=\{R, I\}$ account for the real and imaginary components of the complex channel gains. Given a pair of transmit-antenna $\left(i_{1}, i_{2}\right)$ for $i_{1}=$ $1,2, \ldots, N_{t}$ and $i_{2}=1,2, \ldots, N_{t}$, we denote by $\Sigma_{\left(i_{1}, i_{2}\right)}^{A B}$ the covariance matrix whose element in the $l_{1}$-th row and $l_{2}$-th column is:

$$
\Sigma_{\left(i_{1}, i_{2}\right)}^{A B}\left(l_{1}, l_{2}\right)=\mathrm{E}\left\{\left(\alpha_{i_{1}, l_{1}}^{A}-\mu_{i_{1}, l_{1}}\right)\left(\alpha_{i_{2}, l_{2}}^{B}-\mu_{i_{2}, l_{2}}\right)^{*}\right\}
$$

with $A=\{R, I\}$ and $B=\{R, I\}$.

Moreover, we have, for $i=1,2, \ldots, N_{t}$ and $l=$ $\left.1,2, \ldots, N_{r}\right)$ :

$$
\left\{\begin{array}{l}
\mathrm{E}\left\{\left(\alpha_{i, l}^{R}-\mu_{i, l}\right)\left(\alpha_{i, l}^{R}-\mu_{i, l}\right)^{*}\right\}=\sigma_{i, l}^{2} \\
\mathrm{E}\left\{\left(\alpha_{i, l}^{I}-\mu_{i, l}\right)\left(\alpha_{i, l}^{I}-\mu_{i, l}\right)^{*}\right\}=\sigma_{i, l}^{2} \\
\mathrm{E}\left\{\left(\alpha_{i, l}^{R}-\mu_{i, l}\right)\left(\alpha_{i, l}^{I}-\mu_{i, l}\right)^{*}\right\}=0
\end{array}\right.
$$

due to the assumption of Rician fading.

\section{SSK-MIMO}

\section{ABEP OVER CORRELATED Rician FADING CHANNELS}

Let $m_{n}$ with $n=1,2, \ldots, N_{t}$ be the transmitted message. The signal received after propagation through the wireless fading channel and impinging upon the $l$-th receive-antenna can be written as follows $\left(l=1,2, \ldots, N_{r}\right)$ :

$$
r_{l}(t)=\tilde{s}_{n, l}(t)+n_{l}(t) \quad \text { if } m_{n} \text { is sent }
$$

where $\tilde{s}_{n, l}(t)=\left(s_{n} \otimes h_{n, l}\right)(t)=\alpha_{n, l} s_{n}\left(t-\tau_{n, l}\right)=$ $\beta_{n, l} \exp \left(j \varphi_{n, l}\right) s_{n}\left(t-\tau_{n, l}\right)$ with $n=1,2, \ldots, N_{t}$ and $l=$ $1,2, \ldots, N_{r}$.

In particular, (4) is a general $N_{t}$-hypothesis detection problem [23, Sec. 7.1], [24, Sec. 4.2, pp. 257] in AWGN, when conditioning upon fading channel statistics. Accordingly, the ML-optimum detector with full Channel State Information (CSI) and perfect time-synchronization at the receiver is as follows [5], [23, Sec. 7.1]:

$$
\hat{m}=\underset{m_{i} \text { for } i=1,2, \ldots, N_{t}}{\arg \max }\left\{D_{i}\right\}
$$

where $D_{i}$ for $i=1,2, \ldots, N_{t}$ are the decision metrics defined 
in what follows:

$$
\begin{aligned}
D_{i} & =\sum_{l=1}^{N_{r}}\left[\operatorname{Re}\left\{\int_{T_{m}} r_{l}(t) \tilde{s}_{i, l}^{*}(t) d t\right\}\right] \\
& -\frac{1}{2} \sum_{l=1}^{N_{r}}\left[\int_{T_{m}} \tilde{s}_{i, l}(t) \tilde{s}_{i, l}^{*}(t) d t\right]
\end{aligned}
$$

If the transmitted message is $m_{n}$, which results in switching on the $n$-th transmit-antenna for data transmission, the receiver will be successful in detecting the transmitted message, i.e., $\hat{m}=m_{n}$, if and only if $\max _{i=1,2, \ldots, N_{t}}\left\{D_{i}\right\}=D_{n}$. Furthermore, let us emphasize that the ML-optimum receiver in (5) satisfies the "conventional" channel assumption in [5], and does not require any normalization of the transmitted signal to ensure correct detection for high SNRs. Using this normalization, which would require perfect knowledge of CSI at the transmitter, would lead to the so-called "constrained" channel assumption in [4], [5]. The ML-optimum detector in (5) can efficiently work without any CSI at the transmitter, while still being unbiased for high SNRs (i.e., the error probability tends to zero when the SNR increases without bound).

Conventional SSK modulation in [4]-[6] implicitly assumes that the signal transmitted by the $n$-th transmit-antenna is $s_{n}(t)=\sqrt{E_{m}} w(t) \forall n=1,2, \ldots, N_{t}$, where $w(\cdot)$ denotes the unit-energy (i.e., $\int_{-\infty}^{+\infty}|w(t)|^{2} d t=1$ ) elementary pulse waveform for each transmission. Accordingly, the signal impinging upon the $l$-th receive-antenna is as follows $(l=$ $\left.1,2, \ldots, N_{r}\right)$ :

$$
r_{l}(t)=\sqrt{E_{m}} \beta_{n, l} \exp \left(j \varphi_{n, l}\right) w(t)+n_{l}(t)
$$

We note that in (7) the channel delays $\tau_{n, l}$ for $n=$ $1,2, \ldots, N_{t}$ and $l=1,2, \ldots, N_{r}$ do not appear explicitly. The reason is that (8) implicitly assumes that: either i) a pure sinusoidal tone is transmitted by each antenna, i.e., $w(t)=1 / T_{m}$, and the propagation delays are embedded, with a slight abuse of notation, into the channel phases, or ii) $\tau_{1,1} \cong \tau_{1,2} \cong \ldots \cong \tau_{N_{t}, N_{r}}$, which is a realistic assumption when the distance between the transmitter and the receiver is much larger than the spacing between the transmit- and receive-antenna, and, to a first-order, the signals transmitted by the antennas differ only in phase [25, Eq. (7.24)], which can be still embedded into the channel phases. In this latter case, the delays can be neglected in the light of the assumption of perfect time-synchronization at the receiver. In Section IV, we will see how the transmitted pulse waveforms and the propagation delays might be exploited to get transmit-diversity gains.

From (7), the decision metrics in (6), when conditioned upon the transmission of message $m_{n}$, i.e., $D_{i \mid m_{n}}$, can be written as follows $\left(n=1,2, \ldots, N_{t}\right.$ and $\left.i=1,2, \ldots, N_{t}\right)$ :

$D_{i \mid m_{n}}=\sum_{l=1}^{N_{r}}\left[\operatorname{Re}\left\{\alpha_{n, l} \alpha_{i, l}^{*}\right\} E_{m}+\beta_{i, l} \sqrt{E_{m}} \tilde{n}_{i, l}-\frac{1}{2} \beta_{i, l}^{2} E_{m}\right]$

where $\tilde{n}_{i, l}=\operatorname{Re}\left\{\int_{T_{m}} n_{l}(t) \exp \left(-j \varphi_{i, l}\right) w^{*}(t) d t\right\}$.

\section{A. The $2 \times N_{r}$ MIMO Case}

1) Conditional Error Probability: Let us consider $N_{t}=$ 2. From the decision rule in (5), the probability of wrong detecting the index of the transmit-antenna, $\mathrm{P}_{\mathrm{E}}(\cdot, \cdot)$, when conditioning upon the channel impulse responses $\mathbf{h}_{1}=$ $\left[h_{1,1}, h_{1,2}, \ldots, h_{1, N_{r}}\right]$ and $\mathbf{h}_{2}=\left[h_{2,1}, h_{2,2}, \ldots, h_{2, N_{r}}\right]$, can be explicitly written as follows:

$$
\begin{aligned}
\mathrm{P}_{\mathrm{E}}\left(\mathbf{h}_{1}, \mathbf{h}_{2}\right) & =\frac{1}{2} \operatorname{Pr}\left\{D_{1 \mid m_{1}}<D_{2 \mid m_{1}}\right\} \\
& +\frac{1}{2} \operatorname{Pr}\left\{D_{2 \mid m_{2}}<D_{1 \mid m_{2}}\right\}
\end{aligned}
$$

After a few algebraic manipulations, $\mathrm{P}_{\mathrm{E}}(\cdot, \cdot)$ in (6) can be written as follows:

$$
\mathrm{P}_{\mathrm{E}}\left(\mathbf{h}_{1}, \mathbf{h}_{2}\right)=\operatorname{Pr}\left\{E_{m} \sum_{l=1}^{N_{r}}\left|\alpha_{2, l}-\alpha_{1, l}\right|^{2}<\tilde{n}\right\}
$$

where we have defined $\tilde{n}=\sum_{l=1}^{N_{r}} \tilde{n}_{l}$ with $\tilde{n}_{l}=$ $2 \beta_{2, l} \sqrt{E_{m}} \tilde{n}_{2, l}-2 \beta_{1, l} \sqrt{E_{m}} \tilde{n}_{1, l}$. In particular, when conditioning upon the fading channel gains, $\tilde{n}$ is a Gaussian distributed RV with zero-mean and variance $\mathrm{E}\left\{\tilde{n}^{2}\right\}=$ $4 N_{0} E_{m} \sum_{l=1}^{N_{r}}\left|\alpha_{2, l}-\alpha_{1, l}\right|^{2}$. Furthermore, we have taken into account that, due to symmetry, $\operatorname{Pr}\left\{D_{1 \mid m_{1}}<D_{2 \mid m_{1}}\right\}=$ $\operatorname{Pr}\left\{D_{2 \mid m_{2}}<D_{1 \mid m_{2}}\right\}$.

From (7), $\mathrm{P}_{\mathrm{E}}(\cdot, \cdot)$ can be readily computed in closed-form as follows [24, Sec. 2.2.2]:

$$
\mathrm{P}_{\mathrm{E}}\left(\mathbf{h}_{1}, \mathbf{h}_{2}\right)=Q\left(\sqrt{\bar{\gamma} \sum_{l=1}^{N_{r}}\left|\alpha_{2, l}-\alpha_{1, l}\right|^{2}}\right)
$$

2) Statistics of the End-to-End SNR: To compute the ABEP, the conditional error probability in (8) needs to be averaged over $\left(\mathbf{h}_{1}, \mathbf{h}_{2}\right)$, i.e., $\mathrm{ABEP}_{2,1}=\mathrm{E}\left\{\mathrm{P}_{\mathrm{E}}\left(\mathbf{h}_{1}, \mathbf{h}_{2}\right)\right\}$. We exploit the Moschopoulos method [17] to get a closed-form expression of the ABEP over generically correlated Rician fading channels. To this end, some preliminary considerations are required. In particular, from Section II-B we have $(i=$ $1,2, \ldots, N_{t}$ and $\left.l=1,2, \ldots, N_{r}\right)$ :

$$
\begin{aligned}
\Xi_{l}^{(2,1)} & =\alpha_{2, l}-\alpha_{1, l}=\left(\alpha_{2, l}^{R}+j \alpha_{2, l}^{I}\right)-\left(\alpha_{1, l}^{R}+j \alpha_{1, l}^{I}\right) \\
& =\left(\alpha_{2, l}^{R}-\alpha_{1, l}^{R}\right)+j\left(\alpha_{2, l}^{I}-\alpha_{1, l}^{I}\right)=\xi_{R, l}^{(2,1)}+j \xi_{I, l}^{(2,1)}
\end{aligned}
$$

where we have defined $\xi_{R, l}^{(2,1)}=\alpha_{2, l}^{R}-\alpha_{1, l}^{R}$ and $\xi_{I, l}^{(2,1)}=\alpha_{2, l}^{I}-$ $\alpha_{1, l}^{I}$.

From the channel model in Section II-B, it can be readily shown that the $2 N_{r} \times 1$ column vector $\boldsymbol{\Xi}^{(2,1)}=$ $\left[\xi_{R, 1}^{(2,1)}, \xi_{R, 2}^{(2,1)}, \ldots, \xi_{R, N_{r}}^{(2,1)}, \xi_{I, 1}^{(2,1)}, \xi_{I, 2}^{(2,1)}, \ldots, \xi_{I, N_{r}}^{(2,1)}\right]^{T}$ is Gaussian distributed with mean vector, $\boldsymbol{\mu}_{\Xi^{(2,1)}}$, and covariance matrix, $\Sigma_{\boldsymbol{\Xi}^{(2,1)}}$, defined in what follows, respectively:

$$
\begin{gathered}
\boldsymbol{\mu}_{\Xi^{(2,1)}}=\left[\left(\boldsymbol{\mu}_{2}^{R}-\boldsymbol{\mu}_{1}^{R}\right)^{T},\left(\boldsymbol{\mu}_{2}^{I}-\boldsymbol{\mu}_{1}^{I}\right)^{T}\right]^{T} \\
\boldsymbol{\Sigma}_{\boldsymbol{\Xi}^{(2,1)}}=\left[\begin{array}{cc}
\boldsymbol{\Sigma}_{\Xi^{(2,1)}}^{R R} & \boldsymbol{\Sigma}_{\Xi^{(2,1)}}^{R I} \\
\left(\boldsymbol{\Sigma}_{\boldsymbol{\Xi}^{(2,1)}}^{R I}\right)^{T} & \boldsymbol{\Sigma}_{\Xi^{(2,1)}}^{I I}
\end{array}\right]
\end{gathered}
$$

where $\boldsymbol{\mu}_{i}^{R}=\boldsymbol{\mu}_{i}^{I}=\left[\mu_{i, 1}, \mu_{i, 2}, \ldots, \mu_{i, N_{r}}\right]^{T}$ for $i=1,2$ are 


$$
\left\{\begin{array}{l}
\Psi_{2,1}=\prod_{h=1}^{L}\left[\frac{\chi_{1}^{(2,1)}}{\chi_{h}^{(2,1)}} \exp \left(-\frac{\kappa_{h}^{(2,1)}}{\chi_{h}^{(2,1)}}\right)\right] \\
\vartheta_{k}^{(2,1)}=\frac{1}{k} \sum_{r=1}^{k}\left\{\sum_{b=1}^{L}\left[v_{r}^{(2,1)}\left(1-\frac{\chi_{1}^{(2,1)}}{\chi_{b}^{(2,1)}}\right)^{r}+\frac{r \chi_{1}^{(2,1)} \kappa_{r}^{(2,1)}}{\left(\chi_{b}^{(2,1)}\right)^{2}}\left(1-\frac{\chi_{1}^{(2,1)}}{\chi_{b}^{(2,1)}}\right)^{r-1}\right] \vartheta_{k-r}^{(2,1)}\right\} \quad k \geq 1
\end{array}\right.
$$

$$
\begin{aligned}
\operatorname{ABEP}_{2,1} & =\int_{0}^{+\infty} Q(\sqrt{\bar{\gamma} \zeta}) f_{\gamma_{2,1}}(\zeta) d \zeta \\
& =\frac{\Psi_{2,1}}{2 \sqrt{\pi}} \sum_{k=0}^{+\infty}\left[\frac{\vartheta_{k}^{(2,1)}}{\Gamma\left(v^{(2,1)}+k\right)} G_{2,2}^{2,1}\left(\frac{\bar{\gamma} \chi_{1}^{(2,1)}}{2} \mid \begin{array}{cc}
1-v^{(2,1)}-k & 1 \\
0 & 1 / 2
\end{array}\right)\right]
\end{aligned}
$$

$N_{r} \times 1$ vectors, and $\boldsymbol{\Sigma}_{\boldsymbol{\Xi}^{(2,1)}}^{A B}$ for $A=\{R, I\}$ and $B=\{R, I\}$ are $N_{r} \times N_{r}$ matrices whose element in the $p$-th row and $q-$ th column is $\Sigma_{\Xi^{(2,1)}}^{A B}(p, q)=\Sigma_{(2,2)}^{A B}(p, q)-\boldsymbol{\Sigma}_{(2,1)}^{A B}(p, q)-$ $\Sigma_{(1,2)}^{A B}(p, q)+\Sigma_{(1,1)}^{A B}(p, q)$ for $p=1,2, \ldots, N_{r}$ and $q=$ $1,2, \ldots, N_{r}$.

3) Moschopoulos Method: From (9)-(11), it follows that $\gamma_{2,1}=\sum_{l=1}^{N_{r}}\left|\alpha_{2, l}-\alpha_{1, l}\right|^{2}$ in (8) is the summation of the absolute square value of $N_{r}$ complex Gaussian RVs, with known mean vector (10) and covariance matrix (11). As a consequence, the MGF, $M_{\gamma_{2,1}}(\cdot)$, and the PDF, $f_{\gamma_{2,1}}(\cdot)$, of $\gamma_{2,1}$ can be computed in closed-form by exploiting the general result in [26, Theorem 1]. In particular, the MGF is equal to [26, Eq. (25)]:

$M_{\gamma_{2,1}}(s)=\prod_{h=1}^{L}\left[\left(1+\chi_{h}^{(2,1)} s\right)^{-v_{h}^{(2,1)}} \exp \left(-\frac{\kappa_{h}^{(2,1)} s}{1+\chi_{h}^{(2,1)} s}\right)\right]$

where $L=2 N_{r}$ and, for $h=1,2, \ldots, L, \chi_{h}^{(2,1)}=$ $2 \lambda_{h}^{(2,1)}, v_{h}^{(2,1)}=1 / 2, \kappa_{h}^{(2,1)}=\left(\eta_{h}^{(2,1)}\right)^{2}, \mathbf{D}^{(2,1)}=$ $\operatorname{diag}\left(\lambda_{1}^{(2,1)}, \lambda_{2}^{(2,1)}, \ldots, \lambda_{L}^{(2,1)}\right)$ is the diagonal matrix of the eigenvalues $\left(\lambda_{1}^{(2,1)}, \lambda_{2}^{(2,1)}, \ldots, \lambda_{L}^{(2,1)}\right)$ of the covariance matrix in (11), $\mathbf{T}^{(2,1)}$ is a $L \times L$ orthogonal matrix containing the orthogonal eigenvectors of the covariance matrix in (11) such that $\boldsymbol{\Sigma}_{\boldsymbol{\Xi}^{(2,1)}}=\mathbf{T}^{(2,1)} \mathbf{D}^{(2,1)}\left(\mathbf{T}^{(2,1)}\right)^{T}$, and $\eta_{h}^{(2,1)}$ is the $h$-th element of the column vector $\boldsymbol{\eta}^{(2,1)}=\left(\mathbf{T}^{(2,1)}\right)^{T} \boldsymbol{\mu}_{\boldsymbol{\Xi}^{(2,1)}}$.

From (12), a closed-form expression of the PDF, $f_{\gamma_{2,1}}(\cdot)$, of $\gamma_{2,1}$ can be obtained from [26, Theorem 1] by using the Moschopoulos method. The final result is as follows:

$$
f_{\gamma_{2,1}}(\zeta)=\Psi_{2,1} \sum_{k=0}^{+\infty}\left[\vartheta_{k}^{(2,1)} \frac{\zeta^{v^{(2,1)}+k-1} \exp \left(-\frac{\zeta}{\chi_{1}^{(2,1)}}\right)}{\left(\chi_{1}^{(2,1)}\right)^{v^{(2,1)}+k} \Gamma\left(v^{(2,1)}+k\right)}\right]
$$

where $\Psi_{2,1}$ and $\vartheta_{k}^{(2,1)}$ are defined in (14) on top of this page, and $v^{(2,1)}=\sum_{h=1}^{L} v_{h}^{(2,1)}, \quad \chi_{1}^{(2,1)}=$ $\min \left\{\chi_{h}^{(2,1)} \mid 1 \leq h \leq L\right\}, \vartheta_{0}^{(2,1)}=1$.

4) $M G F-$ and PDF-based Approach to Compute the ABEP: Finally, by using (12) and (13), the $\mathrm{ABEP}_{2,1}$ can be computed from (8) by using either a MGF- or a PDF-based approach [23], as shown in (15) below and in (16) on top of this page, respectively:

$$
\mathrm{ABEP}_{2,1}=\frac{1}{\pi} \int_{0}^{\pi / 2} M_{\gamma_{2,1}}\left(\frac{\bar{\gamma}}{2 \sin ^{2}(\theta)}\right) d \theta
$$

where the last equality in (16) is obtained from the MellinBarnes theorem in [22, Eq. (2.24.1.1)].

Both formulas in (15) and (16) offer the same result. However, the integral in (15) cannot, to the best of our knowledge, be computed in closed-form and numerical methods need to be used. This can be done with consolidated numerical methods, for example Gauss-Legendre Quadrature Rules [27]. On the contrary, (16) offers a truly closed-form expression of the ABEP. An efficient method to compute the series in (13) and (16) can be found in [28].

\section{B. The $N_{t} \times N_{r}$ MIMO Case}

The extension of the framework in Section III-A is quite cumbersome when $N_{t}>2$. To avoid this complexity, we take advantage of a recently proposed bound to compute the performance of SSK modulation when $N_{t}>2$. The bound has been proposed in [10] for a system setup with a single receiveantenna and validated for Nakagami- $m$ fading. In [10], it has been shown that the bound can overcome the limitations of other bounds already available in the literature for Rayleigh fading, e.g., [6]. In this paper, we simply use this bound for a generic MIMO system. The interested reader can find all the details of the derivation in [10].

By using [10, Eq. (33)], the ABEP of a generic $N_{t} \times N_{r}$ SSK-MIMO system over correlated Rician fading channel can be tightly upper-bounded as follows:

$$
\mathrm{ABEP} \leq \mathrm{ABEP}^{\mathrm{B}}=\frac{1}{N_{t}-1} \sum_{i_{1}=1}^{N_{t}} \sum_{i_{2}=i_{1}+1}^{N_{t}} \mathrm{ABEP}_{i_{2}, i_{1}}
$$

where $\mathrm{ABEP}_{i_{2}, i_{1}}$ for $i_{1}=1,2, \ldots, N_{t}$ and $i_{2}=1,2, \ldots, N_{t}$ is the ABEP of an equivalent $2 \times N_{r}$ SSK-MIMO system, which consists of only two transmit-antenna, i.e., the antennas with indexes $i_{1}$ and $i_{2}$. In practice, $\operatorname{ABEP}_{i_{2}, i_{1}}$ for $i_{1}=$ $1,2, \ldots, N_{t}$ and $i_{2}=1,2, \ldots, N_{t}$ can be obtained from (15) and (16) as if the transmit-antenna $i_{1}$ and $i_{2}$ were the only available in the MIMO system. Apart from replacing all the indexes in Section III-A, i.e., $(2,1) \rightarrow\left(i_{2}, i_{1}\right)$, all the formulas are still valid and can be readily applied.

In Section V, we will show that the bound in (17), as based on union bound methods [10], is asymptotically tight for high 
SNRs and can offer good estimate of the ABEP for the SNRs of practical interest.

\section{Analysis of the Diversity Order}

The diversity order of a generic $N_{t} \times N_{r}$ SSK-MIMO system can be computed by using the unifying parametrization in [29]. For example, by using [29, Proposition 3], which applies to the MGF of the end-to-end SNR when $|s| \rightarrow+\infty$, we can readily compute the diversity gain, $G_{d}$, as follows $G_{d}=\sum_{h=1}^{L} v_{h}^{(\cdot, \cdot)}=N_{r}$. Note that, this result is valid for the scenario with arbitrary transmit-antenna, since the MGF when $N_{t}>2$ is simply the summation of the MGFs of equivalent SSK-MIMO systems with two transmit-antenna: this follows from (15) and (17). As obtained in [6] for i.i.d. Rayleigh fading, the diversity gain depends only on the number of receive-antenna, while the number of transmit-antenna affects the coding gain only. The coding gain can be readily computed by using the parametrization in [29] as well. However, it is here omitted due to space constrains.

\section{TOSD-SSK-MIMO:}

\section{ABEP OVER CORRELATED RICIAN FADING CHANNELS}

In Section III-C, we have seen that conventional SSKMIMO systems can offer a diversity gain that depends on only the number of receive-antenna. The aim of this section is to develop a new SSK concept with a higher diversity gain, and, so, with a better ABEP. Unlike conventional SSK modulation that exploits neither the shape of the transmitted signal nor the propagation delays along the different transmit-receive wireless links, we will take advantage of these extra degrees of freedom for system optimization. Furthermore, the proposed scheme will retain the main benefit of SSK from the point of view of the complexity of implementation: a unique transmitantenna is allowed to radiate power at any time instance. This makes the proposed idea fundamentally different from other methods already available to get transmit-diversity gains [20]. Further comments about that are delayed to Section IV-D.

\section{A. Orthogonal Signal Design}

The new SSK-MIMO scheme retains the same MLoptimum detector as conventional SSK modulation, and differs on only the transmitted pulse waveforms. Accordingly, (4)(6) are still valid. However, unlike Section III, the transmitted signal is not simply chosen as $s_{n}(t)=\sqrt{E_{m}} w(t)$ for $n=$ $1,2, \ldots, N_{t}$. The rational and design guideline we adopt to choose $s_{n}(\cdot)$ for $n=1,2, \ldots, N_{t}$ is based on the simple observation as follows. From (8) and (17), we can readily argue that the ABEP depends on the difference of the complex channel gains between pairs of transmit-antenna: the closer the channel gains are, the worse the ABEP is. This result clearly showcases that conventional SSK-MIMO schemes are unable to get diversity from the transmit-antenna, and the motivation is as follows: the complex channel gains in (8) do not sum up in power, but the end-to-end SNR is given by the coherent summation of them, which depends on the constructive and destructive combinations of the channel phases. The main objective of the new SSK-MIMO scheme is to avoid this coherent summation and to allow the complex channel gains in (8) to sum up incoherently, i.e., in power. To achieve this goal, we propose a new method that relies on transmitting signals with good time-correlation properties. For this reason, the new method is called TOSD-SSK.

In formulas, we assume that the signals, $s_{n}(t)=$ $\sqrt{E_{m}} w_{n}(t)$ for $n=1,2, \ldots, N_{t}$, transmitted by each transmit-antenna (when switched on for transmission) satisfy the criterion as follows:

$$
\begin{aligned}
\int_{-\infty}^{+\infty} w_{i_{1}}(t-u) & w_{i_{2}}^{*}(t-v) d t \\
= & \left\{\begin{array}{ll}
1 & \text { if } i_{1}=i_{2} \\
0 & \text { otherwise }
\end{array} \text { and } u=v\right.
\end{aligned}
$$

where the condition needs to be satisfied for $i_{1}=1,2, \ldots, N_{t}$, $i_{2}=1,2, \ldots, N_{t},-\infty \leq u \leq+\infty$, and $-\infty \leq v \leq+\infty$. In other words, the signals $w_{n}(\cdot)$ for $n=1,2, \ldots, N_{t}$ need to have very good cross- and auto-correlation properties in the time domain.

As better described in Section IV-D, the TOSD-SSK modulation scheme can work when the signals have just either good cross- or good auto-correlation properties. In the next sections, we show that with the signal design in (18) we can get some diversity gains from the transmit-antenna. The reader can find in Section IV-D some possibilities for implementation, the limitations of the proposed method, as well as similarities and differences with other state-of-the-art transmit-diversity schemes for MIMO systems.

\section{B. Computation of the ABEP}

Let $s_{n}(t)=\sqrt{E_{m}} w_{n}(t)$ be the transmitted signals for $n=$ $1,2, \ldots, N_{t}$. Then, the signal impinging upon the $l$-th receiveantenna can be written as follows $\left(l=1,2, \ldots, N_{r}\right)$ :

$$
r_{l}(t)=\sqrt{E_{m}} \beta_{n, l} \exp \left(j \varphi_{n, l}\right) w_{n}\left(t-\tau_{n, l}\right)+n_{l}(t)
$$

where we note that, with respect to (7), the channel delays $\tau_{n, l}$ for $n=1,2, \ldots, N_{t}$ and $l=1,2, \ldots, N_{r}$ appear explicitly and might be exploited as described in Section IV-D.

From the new received signal in (19), the decision metrics in (6), when conditioned upon the transmission of message $m_{n}$, i.e., $D_{i \mid m_{n}}$, can be written as follows $\left(n=1,2, \ldots, N_{t}\right.$ and $i=1,2, \ldots, N_{t}$ ):

$$
D_{i \mid m_{n}}=\sum_{l=1}^{N_{r}}\left[\beta_{i, l}^{2} E_{m} \delta_{i, n}+\beta_{i, l} \sqrt{E_{m}} \tilde{n}_{i, l}-\frac{1}{2} \beta_{i, l}^{2} E_{m}\right]
$$

where $\tilde{n}_{i, l}=\operatorname{Re}\left\{\int_{T_{m}} n_{l}(t) \exp \left(-j \varphi_{i, l}\right) w_{i}^{*}\left(t-\tau_{i, l}\right) d t\right\}$.

By comparing (8) and (20), we notice that, due to the orthogonal signal design in (18), we can completely avoid the term $\operatorname{Re}\left\{\alpha_{n, l} \alpha_{i, l}^{*}\right\}$ in (8), which is responsible for the coherent summation of the complex channel gains.

From (20), we can use analytical steps similar to Section III-A and Section III-B to compute the ABEP. Since the analytical development is very similar, we avoid the details of the derivation, and just report the main results. In particular, 
the error probability conditioned upon fading channel statistics is as follows:

$$
\begin{aligned}
& \operatorname{ABEP}\left(\mathbf{h}_{1}, \mathbf{h}_{2}, \ldots, \mathbf{h}_{N_{t}}\right) \leq \operatorname{ABEP}^{\mathrm{B}}\left(\mathbf{h}_{1}, \mathbf{h}_{2}, \ldots, \mathbf{h}_{N_{t}}\right) \\
& =\frac{1}{N_{t}-1} \sum_{i_{1}=1}^{N_{t}} \sum_{i_{2}=i_{1}+1}^{N_{t}} Q\left(\sqrt{\bar{\gamma} \sum_{l=1}^{N_{r}}\left(\left|\alpha_{i_{1}, l}\right|^{2}+\left|\alpha_{i_{2}, l}\right|^{2}\right)}\right)
\end{aligned}
$$

where $\mathbf{h}_{i}=\left[h_{i, 1}, h_{i, 2}, \ldots, h_{i, N_{r}}\right]$ for $i=1,2, \ldots, N_{t}$.

By comparing (8) and (17) with (21), the main advantage of the TOSD-SSK modulation scheme becomes apparent: the end-to-end SNR does not depend on the differences of complex Gaussian RVs, but it is given by their incoherent summation. We will show in Section V that this introduces a significant boost in performance.

From (21), the ABEP can still be computed by exploiting the Moschopoulos method [17] and, in particular, [26, Theorem 1]. As a matter of fact, $\gamma_{i_{2}, i_{1}}=\sum_{l=1}^{N_{r}}\left(\left|\alpha_{i_{1}, l}\right|^{2}+\left|\alpha_{i_{2}, l}\right|^{2}\right)$ is still given by the summation of the absolute square value of $2 N_{r}$ complex Gaussian RVs. Accordingly, (12)-(17) can still be used but for a different mean vector and covariance matrix in (10) and (11), respectively. In particular, let us define the $4 N_{r} \times 1$ vector $\boldsymbol{\Xi}^{\left(i_{2}, i_{1}\right)}=\left[\left(\boldsymbol{\Xi}_{R}^{\left(i_{2}, i_{1}\right)}\right)^{T},\left(\boldsymbol{\Xi}_{I}^{\left(i_{2}, i_{1}\right)}\right)^{T}\right]^{T}$ with $\boldsymbol{\Xi}_{R}^{\left(i_{2}, i_{1}\right)}=$ $\left[\alpha_{i_{1}, 1}^{R}, \alpha_{i_{1}, 2}^{R}, \ldots, \alpha_{i_{1}, N_{r}}^{R}, \alpha_{i_{2}, 1}^{R}, \alpha_{i_{2}, 2}^{R}, \ldots, \alpha_{i_{2}, N_{r}}^{R}\right]^{T} \quad$ and $\boldsymbol{\Xi}_{I}^{\left(i_{2}, i_{1}\right)}=\left[\alpha_{i_{1}, 1}^{I}, \alpha_{i_{1}, 2}^{I}, \ldots, \alpha_{i_{1}, N_{r}}^{I}, \alpha_{i_{2}, 1}^{I}, \alpha_{i_{2}, 2}^{I}, \ldots, \alpha_{i_{2}, N_{r}}^{I}\right]^{T}$ for $i_{1}=1,2, \ldots, N_{t}$ and $i_{2}=1,2, \ldots, N_{t}$. The new $4 N_{r} \times 1$ mean vector, $\boldsymbol{\mu}_{\Xi^{\left(i_{2}, i_{1}\right)}}$, and $4 N_{r} \times 4 N_{r}$ covariance matrix, $\boldsymbol{\Sigma}_{\boldsymbol{\Xi}^{\left(i_{2}, i_{1}\right)}}$, of $\boldsymbol{\Xi}^{\left(i_{2}, i_{1}\right)}$ can be readily computed as follows, respectively:

$$
\begin{gathered}
\boldsymbol{\mu}_{\Xi^{\left(i_{2}, i_{1}\right)}}=\left[\left(\boldsymbol{\mu}_{i_{1}}^{R}\right)^{T},\left(\boldsymbol{\mu}_{i_{2}}^{R}\right)^{T},\left(\boldsymbol{\mu}_{i_{1}}^{I}\right)^{T},\left(\boldsymbol{\mu}_{i_{2}}^{I}\right)^{T}\right]^{T} \\
\boldsymbol{\Sigma}_{\boldsymbol{\Xi}^{\left(i_{2}, i_{1}\right)}}=\left[\begin{array}{cccc}
\boldsymbol{\Sigma}_{i_{1}, i_{1}}^{R R} & \boldsymbol{\Sigma}_{i_{1}, i_{2}}^{R R} & \boldsymbol{\Sigma}_{i_{1}, i_{1}}^{R I} & \boldsymbol{\Sigma}_{i_{1}, i_{2}}^{R I} \\
\left(\boldsymbol{\Sigma}_{i_{1}, i_{2}}^{R R}\right)^{T} & \boldsymbol{\Sigma}_{i_{2}, i_{2}}^{R R} & \left(\boldsymbol{\Sigma}_{i_{1}, i_{2}}^{R I}\right)^{T} & \boldsymbol{\Sigma}_{i_{2}, i_{2}}^{R I} \\
\left(\boldsymbol{\Sigma}_{i_{1}, i_{1}}^{R I}\right)^{T} & \boldsymbol{\Sigma}_{i_{1}, i_{2}}^{R I} & \boldsymbol{\Sigma}_{i_{1}, i_{1}}^{I I} & \boldsymbol{\Sigma}_{i_{1}, i_{2}}^{I I} \\
\left(\boldsymbol{\Sigma}_{i_{1}, i_{2}}^{R I}\right)^{T} & \left(\boldsymbol{\Sigma}_{i_{2}, i_{2}}^{R I}\right)^{T} & \left(\boldsymbol{\Sigma}_{i_{1}, i_{2}}^{I I}\right)^{T} & \boldsymbol{\Sigma}_{i_{2}, i_{2}}^{I I}
\end{array}\right]
\end{gathered}
$$

where $\boldsymbol{\mu}_{i}^{R}=\boldsymbol{\mu}_{i}^{I}=\left[\mu_{i, 1}, \mu_{i, 2}, \ldots, \mu_{1, N_{r}}\right]^{T}$ for $i=$ $1,2, \ldots, N_{t}$.

In summary, also for the TOSD-SSK modulation scheme, we have provided a closed-form framework to compute the ABEP over generically and non-identically distributed Rician fading channels. The ABEP can be computed from (12)-(16) and (21)-(23) by setting $L=4 N_{r}$. All the other symbols and definitions remain the same.

\section{Analysis of the Diversity Order}

The diversity order of the TOSD-SSK modulation scheme can be computed by using a procedure similar to Section III-C. Since for SSK modulation we have $L=2 N_{r}$ and for TOSD-SSK modulation we have $L=4 N_{r}$, it can be readily obtained that the diversity gain is, in the latter case,
$G_{d}=\sum_{h=1}^{L} v_{h}^{(\cdot, \cdot)}=2 N_{r}$. This results in a twofold increase in the diversity order for arbitrary transmit- and receiveantenna. The net effect is a steeper slope of the ABEP for high SNRs and, so, better performance. In particular, when $N_{t}=2$ we can achieve full-diversity, i.e., $G_{d}=2 N_{r}=N_{t} N_{r}$. Further comments about the reason why full-diversity cannot be achieved when $N_{t}>2$ can be found in Section IV-D.

\section{TOSD-SSK: Possibilities of Implementation and Limita- tions}

The aim of this section is to briefly summarize the different ways the TOSD-SSK modulation scheme could be implemented by exploiting a time-orthogonal design of the signals emitted by each transmit-antenna. In particular, some options are as follows.

a) Case Study 1: The first possibility to satisfy the condition in (18) is to assume that the signals transmitted by each transmit-antenna, when switched on for data transmission, are all different from each other and have good cross-correlation properties $^{2}$. This reduces to shape the waveform transmitted by each antenna with a different filter by meeting some timeduration and bandwidth constraints. This way, the information sent by each antenna is conveyed by both the spatial position and the unique pulse shape linked to it.

Using an orthogonal signal design, across the transmitantenna, to get transmit-diversity gain is certainly not new. Several examples can be found in [20, Sec. III]. Probably, the method closest to the scheme suggested here is [30]. However, the TOSD-SSK scheme is different from all the transmit-diversity methods available in the literature for a main reason: in TOSD-SSK there is only a single antenna ${ }^{3}$ transmitting at any time instance [20, Sec. III]. In other words, the proposed method does not achieve transmit-diversity gains due to the simultaneous transmission of redundant signals from several antennas at the transmitter, but because the information is encoded into the spatial position of the transmit-antenna, which is exploited at the receiver via a ML detector. This has a tremendous impact on the complexity of the system: i) inter-antenna synchronization is not required, ii) only a single radio frequency chain at the transmitter is needed, and iii) the receiver exploits a simple single-stream ML detector with linear processing. This makes TOSD-SSK modulation entirely different from similar orthogonal designs for transmitdiversity.

b) Case Study 2: The second choice for satisfying (18) is to assume that the signals transmitted by each transmitantenna, when switched on for data transmission, are delayed versions of a same signal with good auto-correlation prop-

\footnotetext{
${ }^{2}$ We here assume that the propagation delays among the transmit-receive wireless links are negligible. If this is not the case, the condition in (18) should take into account also the propagation delays by means of a feedback channel.

${ }^{3}$ Note that, as described in detail in [6], SSK modulation and transmitantenna selection methods are very different from each other, even though in both cases there is a single (or a sub-set of) active transmit-antenna at any time instance.
} 
erties at those time lags ${ }^{4}$. This reduces to the design of a single waveform with the desired time-duration and bandwidth constraints. This way, the information sent by each antenna is conveyed only by its spatial position.

Broadly speaking, this technique is not new in the MIMO context and is known as delay-diversity method. The first paper proposing the idea was [31]. Other examples can be found in [20, Sec. III]. However, similar to Case Study 1, all the schemes based on this method foresee to artificially create multipath distortion via the simultaneous transmission of redundant signals from several transmit-antenna. So, the proposed method is substantially different from them since it exploits the space modulation concept to allow a single antenna to be active at any time instance. The benefits already described for Case Study 1 are retained by this implementation as well.

c) Case Study 3: A slight different alternative to Case Study 2 is to consider that all the transmit-antenna emit exactly the same signal (instead of delayed versions of it), which should have good auto-correlation properties at all time lags. The idea of this implementation is to exploit the different propagation delays along any transmit-receive wireless link. In other words, while conventional SSK modulation does not exploit the different propagation delays along the different wireless links (this is achieved by assuming, e.g., that these delays are negligible or by transmitting a single sinusoidal pulse that allows to map the time delays into actual phase shifts [10], [11]), the TOSD-SSK scheme could take advantage of them. By transmitting a signal with very good auto-correlation properties (ideally a Dirac delta pulse) all the delays could be resolved in time. Of course, the effectiveness of this solution depends on the spacing of the antenna elements (at both the transmitter and the receiver) and the characteristics of the wireless channel. This solutions may be certainly effective in a distributed setting, by applying the SM concept to virtual MIMO systems. The constraints on the signal design of this solution might also be relaxed in part by assuming the presence of a feedback channel from the receiver to the transmitter: the receiver might estimate the time lags over the transmit-receive wireless links and feedback them to the transmitter. With this information, the transmitter might design a pulse shape with good auto-correlation properties for those time delays only.

d) Case Study 4: Finally, an effective way to exploit the TOSD-SSK method is to rely on the good auto- and crosscorrelation properties of Spread Spectrum (SS) and Ultra Wide Band (UWB) signals. Using SS signals for transmit-diversity gains is not new [32]. However, in [32] all the antennas are still required to transmit redundant signals simultaneously, which does not make this solution suitable for low-complexity implementations. To our best knowledge, no solutions are available in the literature that rely on the UWB signaling to get transmit-diversity gains. However, UWB signals might be a very appealing solution for SSK modulation [6], and the accurate ranging and synchronization capabilities of Impulse

\footnotetext{
${ }^{4}$ We here assume that the propagation delays among the transmit-receive wireless links are negligible. If this is not the case, the condition in (18) should take into account also the propagation delays by means of a feedback channel.
}

Radio (IR-) UWB [33] could be a viable choice to exploit the TOSD-SSK method based on the design of a peaky auto-correlation function. However, a sound assessment of the IR-UWB technology for this purpose requires to extend the system model to a frequency-selective fading channel. This is out of the scope of this paper and is left to a future contribution.

In summary, the proposed TOSD-SSK modulation scheme can be implemented in several ways and appears to be fundamentally different from other transmit-diversity schemes: it exploits the space modulation concept to get transmit-diversity gains and does not rely on the transmission of redundant copies of the same signal. This seems to be suitable for lowcomplexity and low-cost MIMO implementations. Moreover, no feedback from the receiver to the transmitter seems to be required for various implementations. However, the main limitation of the proposed approach, if compared to more complicated methods, e.g., [19], is the impossibility to achieve full-diversity when $N_{t}>2$. The reason is that the TOSD-SSK principle has been conceived from the analytical framework in (21), which can capture only in part the system behavior since it is obtained by using union bound methods. Current research is now devoted to develop other analytical models, from which more insightful information about the system optimization can be obtained. However, this is left to a future contribution.

In addition, let us emphasize that in this paper we are not interested in the specific details related to each possible implementation to satisfying the optimization criterion in (18). As a matter of fact, our goal is to propose the main optimization criterion to get transmit-diversity gains and compute the achievable performance under ideal conditions, which provide a strict lower-bound of the performance that can be expected by the TOSD-SSK modulation scheme for a practical operational design. On the other hand, in Section $\mathrm{V}$ we need a practical way to implement the TOSD-SSK modulation concept in order to obtain the numerical results and compare them with our analytical derivation. Several ways to design orthogonal signals can, e.g., be found in [34]. In particular, as far as the TOSD-SSK modulation scheme is concerned, we have found particularly suitable an orthogonal signal design method that has been recently proposed for UWB wireless communication systems. However, the approach is not restricted to signals with a very large bandwidth, but can be used for narrow-band signals as well. The idea can be found in [35], and is introduced in Section V.

Finally, we would like to emphasize that the TOSD-SSK modulation concept introduces some additional practical design issues with respect to the SSK modulation scheme. Some of these issues are specific to the particular method used to achieve the time-orthogonal signal design in (18). For example, as mentioned above, Case Study 3 might require a feedback channel and Case Study 4 should take into account the large bandwidth requirements of SS and UWB signals, along with frequency distortions possibly introduced by the wireless channel for these transmission schemes. In general, practical issues to be considered when designing orthogonal signals according to (18) are, among many others: i) the achievable duration/bandwidth product of the designed waveforms, ii) the 
amount of channel state information needed at the transmitter to guaranteeing the orthogonality at the receiver, which may change depending on the specific channel model, iii) the availability and properties of the feedback channel to ensure the reliable transmission of channel state information, and iv) the synchronization requirements at the transmitter and at the receiver to guaranteeing the orthogonality constraint. However, the analysis of each of these issues is beyond the scope of this paper and is postponed to a future research contribution. In Section V, we show that the practical solution adopted in this paper for illustrative purposes [35], only requires, similar to the SSK modulation scheme, perfect time-synchronization at the receiver. Moreover, both SSK and TOSD-SSK modulation schemes offer the same spectral efficiency, which allows us to perform a fair performance comparison.

\section{Numerical And Simulation Results}

In this section, we describe some numerical results aiming at validating the accuracy of the proposed analytical frameworks, at comparing the performance of SSK and TOSD-SSK modulation schemes, and at substantiating the claims that have been made in the sections above.

a) System Setup: For illustrative purposes, the following setup is used. i) The curves are shown as a function of the Rice factor $K_{R}^{(i, l)}$ and the average SNR of the transmit-receive wireless link from the $i$-th transmitantenna to the $l$-th receive-antenna for $i=1,2, \ldots, N_{t}$ and $l=1,2, \ldots, N_{r}$. The parameters (i.e., mean and standard deviation) of the related Gaussian RVs can be computed as (see Section II-B) $\mu_{i, l}=\sqrt{\Omega_{i, l}\left[K_{R}^{(i, l)} /\left(K_{R}^{(i, l)}+1\right)\right]}$ and $\sigma_{i, l}=\sqrt{\Omega_{i, l} /\left[2\left(K_{R}^{(i, l)}+1\right)\right]}$ for $i=1,2, \ldots, N_{t}$ and $l=1,2, \ldots, N_{r}$. ii) As far as the channel correlation model is concerned, we consider the "Kronecker model" [36], [37] with exponential correlation [38]. However, we emphasize here that the analytical frameworks proposed in Section III and in Section IV can be used for arbitrary correlation matrices, including correlation matrices obtained from measurements. The adoption of a "Kronecker model" is only due to its simplicity of implementation, and we expect that SSK modulation will show similar performance trends for different correlation models. In particular, we assume ${ }^{5}$ $\Sigma_{i_{1}, i_{2}}^{R R}\left(l_{1} . l_{2}\right)=\Sigma_{i_{1}, i_{2}}^{I I}\left(l_{1} . l_{2}\right)=\sigma_{i_{1}, l_{1}} \sigma_{i_{2}, l_{2}} \rho^{\left|i_{1}-i_{2}\right|} \rho^{\left|l_{1}-l_{2}\right|}$ and $\Sigma_{i_{1}, i_{2}}^{R I}\left(l_{1} \cdot l_{2}\right)=\Sigma_{i_{1}, i_{2}}^{I R}\left(l_{1} \cdot l_{2}\right)=0$ for $i_{1}=1,2, \ldots, N_{t}$, $i_{2}=1,2, \ldots, N_{t}, l_{1}=1,2, \ldots, N_{r}$, and $l_{2}=1,2, \ldots, N_{r}$. Furthermore, $\rho \in[0,1]$ is a constant factor used to study the performance for various correlation coefficients. iii) The numerical values of all the parameters can be found in the caption of each figure.

As far as Monte Carlo simulations are concerned, we have implemented the detectors in (5) and (6) for SSK modulation and added the constraint in (18) for TOSD-SSK modulation. For each transmitted symbol, which is composed by $\log _{2}\left(N_{t}\right)$ bits, we assume the wireless channel to be constant, while it

\footnotetext{
${ }^{5}$ Note that with this notation we implicitly assume, with a slight abuse of notation, $\rho^{0}=1$ for all the values of $\rho$.
}

changes independently from symbol to symbol according to the fading channel model described above. To implement the TOSD-SSK concept in practice, i.e., to obtain a set of signal waveforms $w_{n}(\cdot)$ for $n=1,2, \ldots, N_{t}$ that satisfy (18), we use the approach proposed in [35]. In particular, in [35] the authors have proposed a general method to design orthogonal waveforms with almost similar time-duration and frequencybandwidth. Although the idea has been proposed for UWB systems, the signal design method in [35] can be used to obtain narrow-band waveforms via simple time-scaling operations. More specifically, if, e.g., $N_{t}=2$, we assume that the first transmit-antenna emits the signal [35, Eq. (38)] and the second transmit-antenna emits the signal [35, Eq. (39)], when they are switched on for transmission. Of course, any other combinations of signals in [35] is possible. This specific implementation reproduces the idea described in Case Study 1 in Section IV-D, since the waveforms in [35] have good crosscorrelation properties ${ }^{6}$. On the other hand, when the SSK modulation scheme is simulated, we consider that each antenna radiates always the same pulse shape, for example the signal [35, Eq. (38)]. With this assumption, both SSK and TOSDSSK modulation schemes use waveforms with a similar timeduration and frequency-bandwidth, thus offering almost the same spectral efficiency [35, Fig. 4 and Fig. 5], and allowing us for a fair comparison. For the sake of simplicity, we assume perfect time-synchronization at the transmitter and at the receiver, as well as that the time propagation delays are almost the same over all the wireless links. These assumptions all agree with the system model in Section II, and allow us to get strict lower-bounds of the ABEP achievable by the SSK and TOSD-SSK modulation schemes. The design of orthogonal waveforms robust to time-asynchronism (also known as jitterrobust signal design) has recently received a growing attention in the literature (see, e.g., [39] and references therein), but due to space constraints the study of this scenario is postponed to future research.

As far as the analytical framework is concerned, in Section III-A.4 we have shown that the ABEP can be computed by using either a MGF- or a PDF-based approach. From a numerical point of view, we have found the MGF-based method to be less computational intensive for increasing values of the correlation coefficient of the wireless channel. So, we have used the PDF-based approach to compute the ABEP for low correlation coefficients (i.e., $\rho \leq 0.25$ ) and the MGFbased approach for high correlation coefficients.

b) Performance of SSK Modulation over Rician Fading: In Figs. 1-4, we show the ABEP of SSK modulation for various combinations of transmit- and receive-antenna over the same Rician fading channel with identically distributed fading. The following trends can be observed. i) We notice that the analytical model developed in Section III is very accurate, and the bound for $N_{t}>2$ tightly overlaps with Monte Carlo simulations for $\mathrm{ABEP} \leq 10^{-1}$, which is the range of interest for practical applications. ii) The ABEP degrades for increasing values of the Rice factor $K_{R}^{(i, l)}=K_{R}$

\footnotetext{
${ }^{6}$ We note that using a pulse-based transmission scheme for SSK modulation might have some implementation benefits, as described in [6].
} 


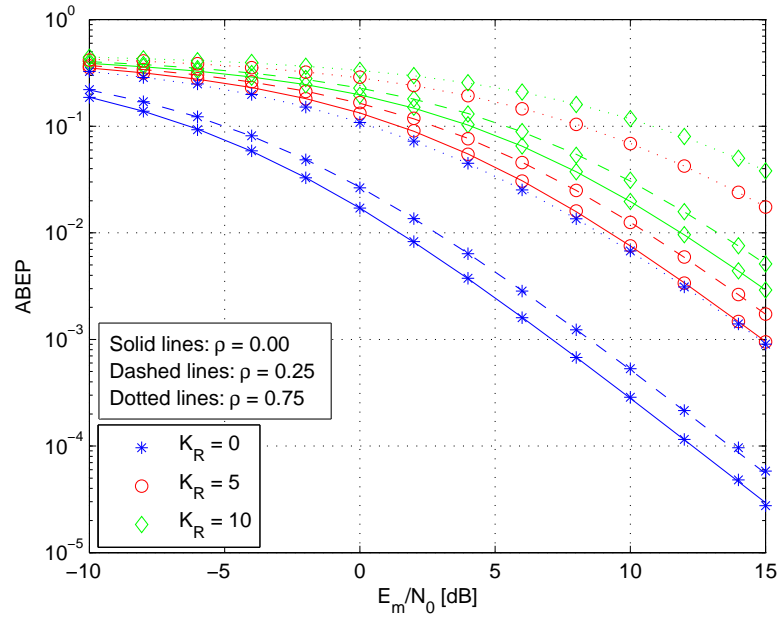

Fig. 1. SSK modulation: ABEP against $E_{m} / N_{0}$. Solid, dashed, and dotted lines denote the analytical model in Section III and markers Monte Carlo simulations. Setup: i) $N_{t}=2$, ii) $N_{r}=2$, iii) $\Omega_{i, l}=10 \mathrm{~dB}$ and $K_{R}^{(i, l)}=$ $K_{R}$ for $i=1,2, \ldots, N_{t}$ and $l=1,2, \ldots, N_{r}$, and iv) $\rho=0.00$ (solid lines), $\rho=0.25$ (dashed lines), $\rho=0.75$ (dotted lines).

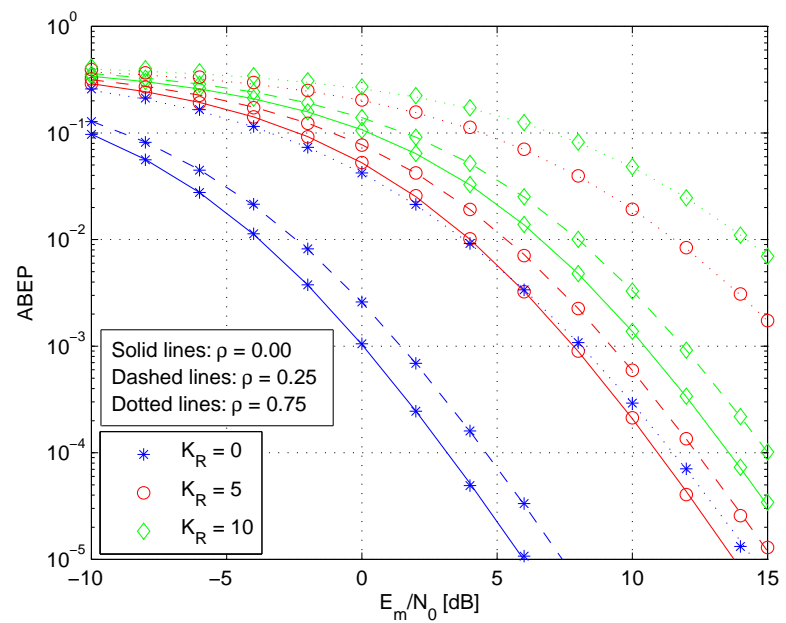

Fig. 2. SSK modulation: ABEP against $E_{m} / N_{0}$. Solid, dashed, and dotted lines denote the analytical model in Section III and markers Monte Carlo simulations. Setup: i) $N_{t}=2$, ii) $N_{r}=4$, iii) $\Omega_{i, l}=10 \mathrm{~dB}$ and $K_{R}^{(i, l)}=$ $K_{R}$ for $i=1,2, \ldots, N_{t}$ and $l=1,2, \ldots, N_{r}$, and iv) $\rho=0.00$ (solid lines), $\rho=0.25$ (dashed lines), $\rho=0.75$ (dotted lines).

for $i=1,2, \ldots, N_{t}$ and $l=1,2, \ldots, N_{r}$. The reason is as follows. For increasing values of $K_{R}$ the fading fluctuations tend to be less pronounced and the amount of fading gets smaller [23]. In particular, we have Rayleigh fading if $K_{R}=0$ and no fading if $K_{R} \rightarrow+\infty$. Since the scenario in Figs. 14 considers a wireless channel with the same average SNR $\Omega_{i, l}=\Omega$ for $i=1,2, \ldots, N_{t}$ and $l=1,2, \ldots, N_{r}$, from (8) and (17) we conclude that lower amounts of fading bring the complex channel gains closer together, which results in a poor ABEP. This is a very different behavior with respect to conventional modulation schemes, where, in general, the ABEP improves for less pronounced fading fluctuations [23]. However, this result is inherent in the working mechanism of SSK modulation, which requires the wireless links to be suffi-

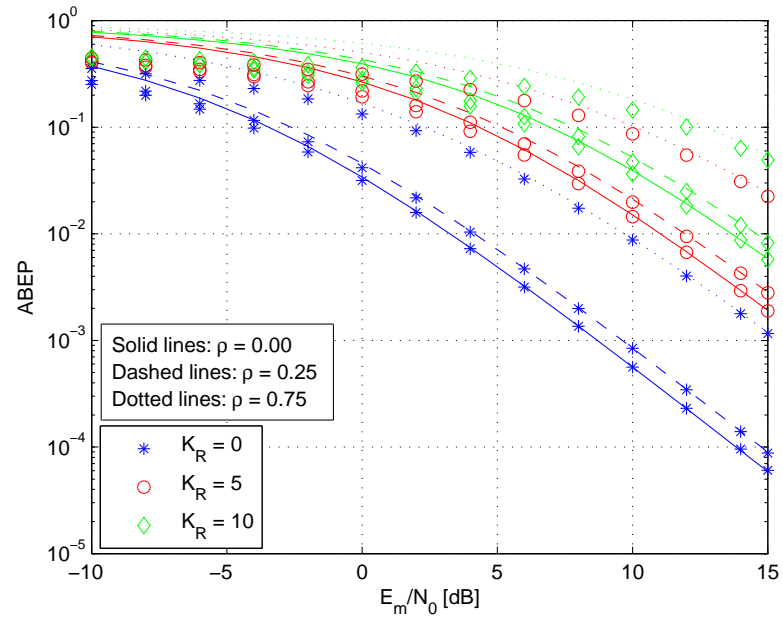

Fig. 3. SSK modulation: ABEP against $E_{m} / N_{0}$. Solid, dashed, and dotted lines denote the analytical model in Section III and markers Monte Carlo simulations. Setup: i) $N_{t}=4$, ii) $N_{r}=2$, iii) $\Omega_{i, l}=10 \mathrm{~dB}$ and $K_{R}^{(i, l)}=$ $K_{R}$ for $i=1,2, \ldots, N_{t}$ and $l=1,2, \ldots, N_{r}$, and iv) $\rho=0.00$ (solid lines), $\rho=0.25$ (dashed lines), $\rho=0.75$ (dotted lines).

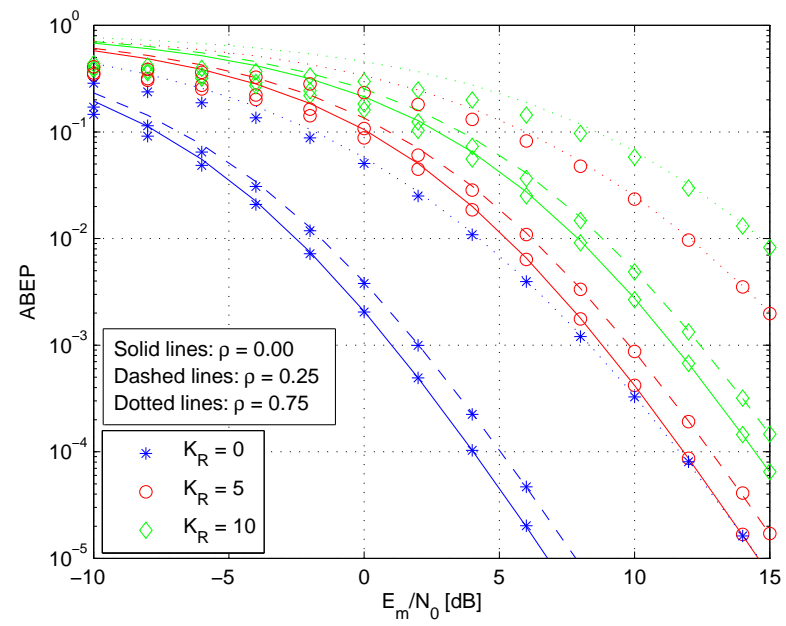

Fig. 4. SSK modulation: ABEP against $E_{m} / N_{0}$. Solid, dashed, and dotted lines denote the analytical model in Section III and markers Monte Carlo simulations. Setup: i) $N_{t}=4$, ii) $N_{r}=4$, iii) $\Omega_{i, l}=10 \mathrm{~dB}$ and $K_{R}^{(i, l)}=$ $K_{R}$ for $i=1,2, \ldots, N_{t}$ and $l=1,2, \ldots, N_{r}$, and iv) $\rho=0.00$ (solid lines), $\rho=0.25$ (dashed lines), $\rho=0.75$ (dotted lines).

ciently different from each other to achieve good performance. iii) By studying the ABEP with respect to the correlation coefficient, we observe that it gets worse for increasing values of the channel correlation, as expected. In particular, we observe that for large values of the correlation coefficient the detector might be unable to distinguish the messages sent by the different transmit-antenna. This conclusion also follows from (8) and (17), similar to the comment in ii). iv) Finally, by studying the performance with respect to the number of transmit- and receive-antenna we observe that the ABEP degrades for increasing values of $N_{t}$, while it gets better for increasing values of $N_{r}$. The trend obtained with respect to $N_{t}$ is similar to the performance degradation observed in digital modulation schemes with increasing values of the modulation 


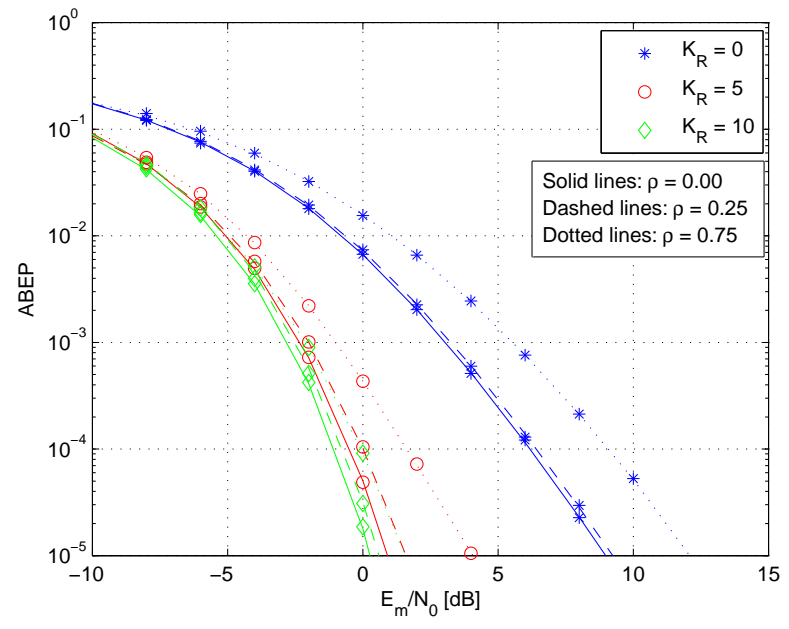

Fig. 5. TOSD-SSK modulation: ABEP against $E_{m} / N_{0}$. Solid, dashed, and dotted lines denote the analytical model in Section IV and markers Monte Carlo simulations. Setup: i) $N_{t}=2$, ii) $N_{r}=2$, iii) $\Omega_{i, l}=10 \mathrm{~dB}$ and $K_{R}^{(i, l)}=K_{R}$ for $i=1,2, \ldots, N_{t}$ and $l=1,2, \ldots, N_{r}$, and iv) $\rho=0.00$ (solid lines), $\rho=0.25$ (dashed lines), $\rho=0.75$ (dotted lines).

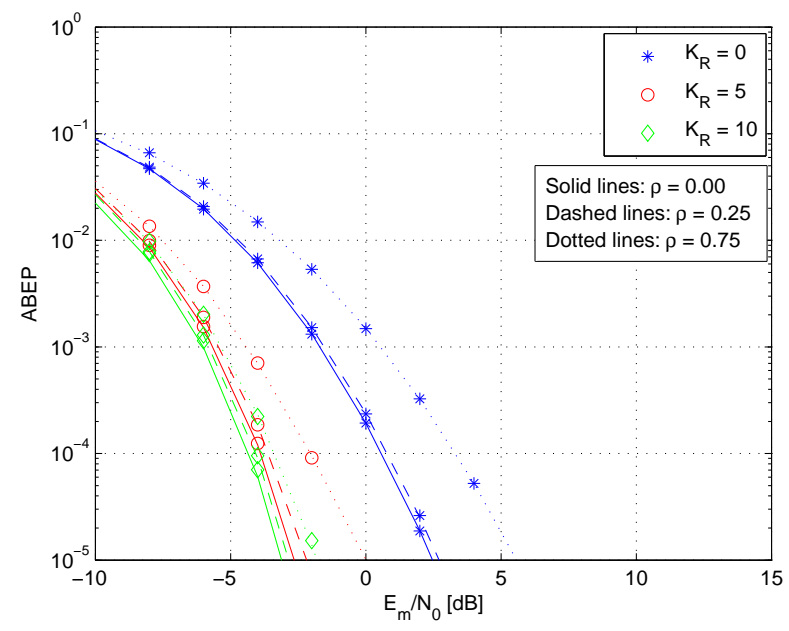

Fig. 6. TOSD-SSK modulation: ABEP against $E_{m} / N_{0}$. Solid, dashed, and dotted lines denote the analytical model in Section IV and markers Monte Carlo simulations. Setup: i) $N_{t}=2$, ii) $N_{r}=4$, iii) $\Omega_{i, l}=10 \mathrm{~dB}$ and $K_{R}^{(i, l)}=K_{R}$ for $i=1,2, \ldots, N_{t}$ and $l=1,2, \ldots, N_{r}$, and iv) $\rho=0.00$ (solid lines), $\rho=0.25$ (dashed lines), $\rho=0.75$ (dotted lines).

order: this agrees with (17). Furthermore, numerical results confirm that the diversity order increases with $N_{r}$, as shown in Section III-C.

c) Performance of TOSD-SSK Modulation over Rician Fading: In Figs. 5-8, we show the ABEP of TOSD-SSK modulation for the same system setup as in Figs. 1-4. Also in this case, we notice that the analytical model developed in Section IV is very accurate and the bound for $N_{t}>2$ tightly overlaps with Monte Carlo simulations for ABEP $\leq 10^{-1}$. However, with respect to Figs. 1-4, we can observe a very different trend of the ABEP with respect to the channel fading, which makes TOSD-SSK more similar to conventional digital modulation schemes. In particular: i) we observe that the ABEP of TOSD-SSK modulation gets better for increasing

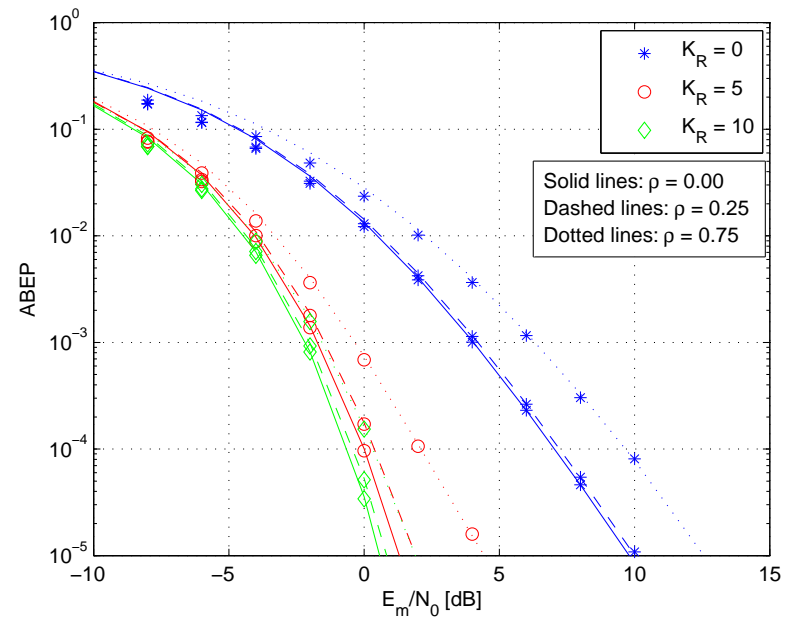

Fig. 7. TOSD-SSK modulation: ABEP against $E_{m} / N_{0}$. Solid, dashed, and dotted lines denote the analytical model in Section IV and markers Monte Carlo simulations. Setup: i) $N_{t}=4$, ii) $N_{r}=2$, iii) $\Omega_{i, l}=10 \mathrm{~dB}$ and $K_{R}^{(i, l)}=K_{R}$ for $i=1,2, \ldots, N_{t}$ and $l=1,2, \ldots, N_{r}$, and iv) $\rho=0.00$ (solid lines), $\rho=0.25$ (dashed lines), $\rho=0.75$ (dotted lines).

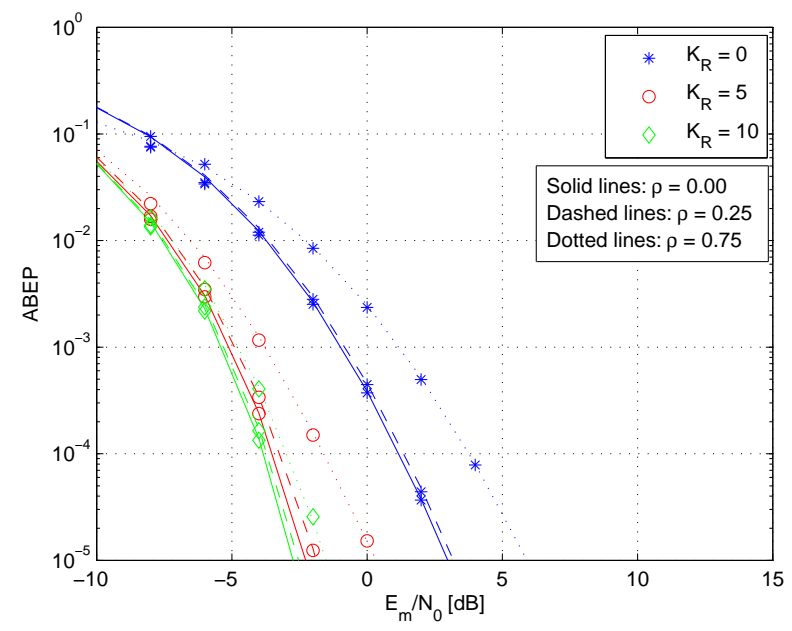

Fig. 8. TOSD-SSK modulation: ABEP against $E_{m} / N_{0}$. Solid, dashed, and dotted lines denote the analytical model in Section IV and markers Monte Carlo simulations. Setup: i) $N_{t}=4$, ii) $N_{r}=4$, iii) $\Omega_{i, l}=10 \mathrm{~dB}$ and $K_{R}^{(i, l)}=K_{R}$ for $i=1,2, \ldots, N_{t}$ and $l=1,2, \ldots, N_{r}$, and iv) $\rho=0.00$ (solid lines), $\rho=0.25$ (dashed lines), $\rho=0.75$ (dotted lines).

values of the Rice factor $K_{R}$ : this stems directly from (21), which clearly showcases that the ABEP does not depend on the difference between the complex channels gains, and, so, on how different the fading on the wireless links is, but it depends on the power-sum of them. This is a behavior similar to conventional modulation schemes with optimum combining [23]; and ii) also in this case the ABEP degrades for increasing values of the correlation coefficient. However, if compared to Figs. 1-4, the degradation of the ABEP is less pronounced. The net result is that TOSD-SSK modulation is inherently more robust to channel correlation than SSK modulation. Finally, similar to Figs. 1-4, the ABEP gets slightly worse for increasing values of $N_{t}$ and significantly better when $N_{r}$ increases. The numerical results confirm that TOSD-SSK 


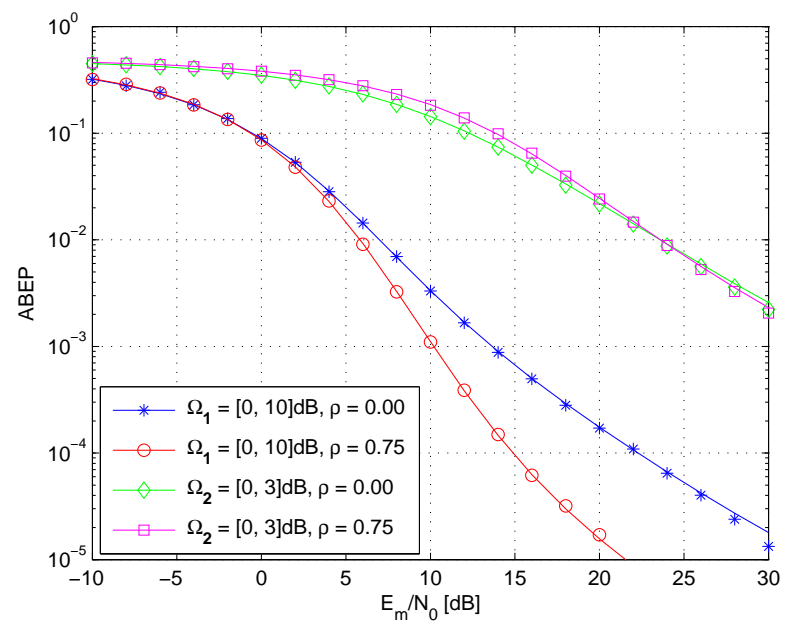

Fig. 9. SSK modulation: ABEP against $E_{m} / N_{0}$. Solid lines denote the analytical model in Section III and markers Monte Carlo simulations. Setup: i) $N_{t}=2$, ii) $N_{r}=1$, iii) $\boldsymbol{\Omega}_{\mathbf{1}}=\left[\Omega_{1,1}, \Omega_{2,1}\right]=[0,10] \mathrm{dB}, \boldsymbol{\Omega}_{\mathbf{2}}=$ $\left[\Omega_{1,1}, \Omega_{2,1}\right]=[0,3] \mathrm{dB}$, and iv) $K_{R}^{(i, l)}=K_{R}=5 \mathrm{~dB}$ for $i=1,2, \ldots, N_{t}$ and $l=1,2, \ldots, N_{r}$.

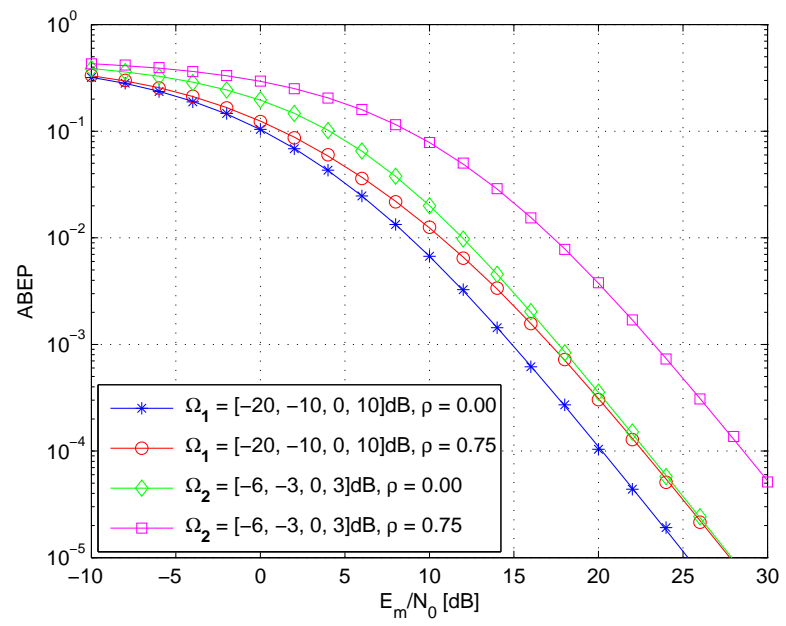

Fig. 10. SSK modulation: ABEP against $E_{m} / N_{0}$. Solid lines denote the analytical model in Section III and markers Monte Carlo simulations. Setup: i) $N_{t}=2$, ii) $N_{r}=2$, iii) $\boldsymbol{\Omega}_{1}=\left[\Omega_{1,1}, \Omega_{1,2}, \Omega_{2,1}, \Omega_{2,2}\right]=$ $[-20,-10,0,10] \mathrm{dB}, \boldsymbol{\Omega}_{\mathbf{2}}=\left[\Omega_{1,1}, \Omega_{1,2}, \Omega_{2,1}, \Omega_{2,2}\right]=[-6,-3,0,3] \mathrm{dB}$, and iv) $K_{R}^{(i, l)}=K_{R}=0 \mathrm{~dB}$ for $i=1,2, \ldots, N_{t}$ and $l=1,2, \ldots, N_{r}$.

modulation offers a diversity order equal to $2 N_{r}$, as shown in Section IV-C.

d) Performance of SSK Modulation over Rician Fading - Power Imbalance: In Figs. 9, 10, we study the performance of SSK modulation by considering a wireless scenario with non-identically distributed fading (i.e., with power imbalance among the wireless links). Due to space constraints, similar curves for TOSD-SSK modulation are not reproduced because, as already observed in Figs. 5-8, TOSD-SSK modulation shows a trend similar to conventional modulation schemes [23]. Besides the good agreement between Monte Carlo simulations and the analytical framework in Section III, we notice that the performance of SSK changes remarkably for various fading scenarios. In particular, in Fig. 9 we observe

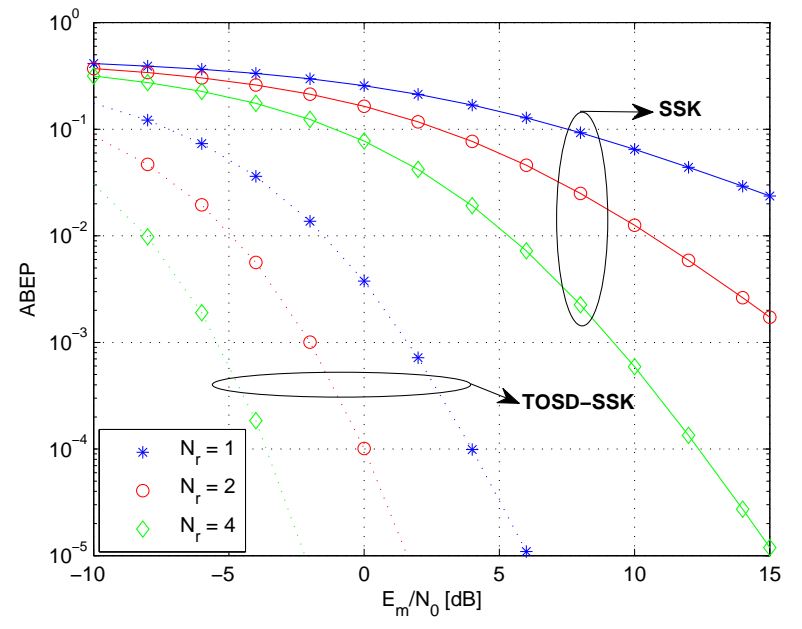

Fig. 11. Comparison between SSK and TOSD-SSK modulation: ABEP against $E_{m} / N_{0}$. Markers with solid lines denote the analytical model in Section III and markers with dotted lines the analytical model in Section IV. Setup: i) $N_{t}=2$, ii) $\Omega_{i, l}=10 \mathrm{~dB}$ and $K_{R}^{(i, l)}=5 \mathrm{~dB}$ for $i=1,2, \ldots, N_{t}$ and $l=1,2, \ldots, N_{r}$, and iii) $\rho=0.25$.

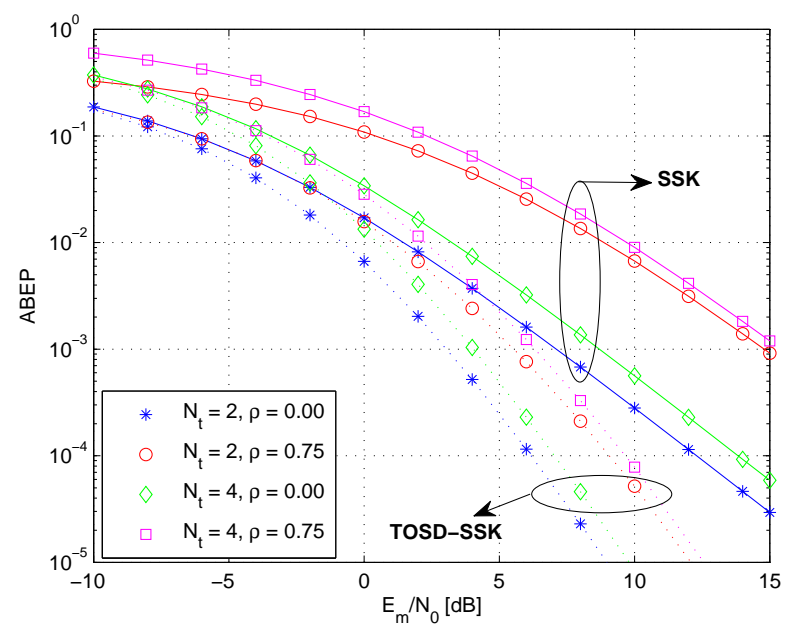

Fig. 12. Comparison between SSK and TOSD-SSK modulation: ABEP against $E_{m} / N_{0}$. Markers with solid lines denote the analytical model in Section III and markers with dotted lines the analytical model in Section IV. Setup: i) $N_{r}=2$, and ii) $\Omega_{i, l}=10 \mathrm{~dB}$ and $K_{R}^{(i, l)}=0 \mathrm{~dB}$ for $i=$ $1,2, \ldots, N_{t}$ and $l=1,2, \ldots, N_{r}$.

two scenarios with a high (i.e, $\Omega_{2,1}-\Omega_{1,1}=10 \mathrm{~dB}$ ) and a low (i.e, $\Omega_{2,1}-\Omega_{1,1}=3 \mathrm{~dB}$ ) power imbalance between the wireless links. We notice that when the fading fluctuations are not very pronounced (i.e., $K_{R}=5 \mathrm{~dB}$ ), the ABEP improves with channel correlation, and the improvement increases with the average power gap $\Omega_{2,1}-\Omega_{1,1}$. This trend follows from (8) and (17): although the channel fading might introduce fluctuations that could offset the power imbalance of the channel gains, if the wireless links are correlated these fluctuations are likely to be almost the same over all the wireless links, thus unlikely offsetting the average power gap. On the contrary, if the wireless links are identically distributed (as shown in Figs. 1-4) channel fading can only be beneficial if the wireless links are more distinguishable from each other, and so, in 
this case, channel correlation can only be detrimental. These conclusions agree with the results already obtained in [10], [11] for Nakagami- $m$ fading, thus showing that this property is inherent in SSK and is not specific of the channel fading model. In Fig. 10, we show a setup similar to Fig. 9, but in this case the channels are subject to more pronounced fading fluctuations (i.e., $K_{R}=0 \mathrm{~dB}$ instead of $K_{R}=5 \mathrm{~dB}$ as in Fig. 9). We notice that in Fig. 10 the ABEP gets worse for increasing values of the channel correlation even though there is a non-negligible power imbalance among the wireless links. The reason is that the deep fluctuations introduced by Rayleigh fading $\left(K_{R}=0 \mathrm{~dB}\right)$ offset the average power gap in this case. However, with respect to Figs. 1-4, we observe that power imbalance reduces, in any case, the effect of fading fluctuations and, so, the ABEP improves. These results clearly show that the performance of SSK over generalized fading channels is very unpredictable, and the development of general and sound frameworks, as those derived in this paper, are very important for the optimization of this novel transmission technique.

e) SSK and TOSD- Modulation over Rician Fading Performance Comparison: In Figs. 11, 12 we compare the performance of SSK and TOSD-SSK modulation over the same wireless scenarios. The interested reader might also find useful to carefully analyze, curve-by-curve, Figs. 1-4 with Figs. 5-8 for a comparison over a wide range of fading parameters and combinations of transmit- and receive-antenna. In particular, Fig. 11 shows the significant performance gain achieved by TOSD-SSK modulation with respect to SSK modulation. We observe that the performance improvement is of the order of tens of $\mathrm{dB}$, and it is mainly due to the steeper slope (higher diversity order) of the ABEP for high SNRs. Finally, in Fig. 12 we observe that TOSD-SSK modulation introduces the same performance drop as SSK modulation for increasing values of $N_{t}$, while it seems to be more robust than SSK modulation to channel correlation, which is an additional benefit when using this technology on mobile handsets.

\section{CONCLUSION}

In this paper, we have studied the performance of SSK modulation over correlated Rician fading channels. An accurate analytical framework has been developed, and numerical results have clearly indicated that the performance of SSK modulation is very unpredictable and strongly depends on the actual channel conditions. Furthermore, we have shown that conventional SSK modulation schemes are unable to offer transmit-diversity gains, which might limit their usefulness in the downlink. Deviating from this limitation, we have proposed a novel SSK modulation scheme, which is called TOSD-SSK and is based on a time-orthogonal design of the transmitted signals. Analysis and simulations have shown that the novel scheme can offer full-diversity for two transmit- and arbitrary receive-antenna, and is more robust than SSK modulation to channel correlation. Ongoing research is concerned with the development of improved SSK modulation schemes offering full-diversity for arbitrary transmit-antenna.

\section{ACKNOWLEDGMENT}

We gratefully acknowledge support from the EPSRC (EP/G011788/1) for this work. Harald Haas acknowledges the Scottish Funding Council support of his position within the Edinburgh Research Partnership in Engineering and Mathematics between the University of Edinburgh and Heriot Watt University.

\section{REFERENCES}

[1] Y. Chau and S.-H. Yu, "Space modulation on wireless fading channels", IEEE Veh. Technol. Conf., vol. 3, pp. 1668-1671, Oct. 2001.

[2] H. Haas, E. Costa, and E. Schultz, "Increasing spectral efficiency by data multiplexing using antennas arrays", IEEE Int. Symp. Personal, Indoor, Mobile Radio Commun., vol. 2, pp. 610-613, Sept. 2002.

[3] Y. Yang and B. Jiao, "Information-guided channel-hopping for high data rate wireless communication", IEEE Commun. Lett., vol. 12, no. 4, pp. 225-227, Apr. 2008.

[4] R. Y. Mesleh, H. Haas, S. Sinanovic, C. W. Ahn, and S. Yun, "Spatial modulation", IEEE Trans. Veh. Technol., vol. 57, no. 4, pp. 2228-2241, July 2008.

[5] J. Jeganathan, A. Ghrayeb, and L. Szczecinski, "Spatial modulation: Optimal detection and performance analysis", IEEE Commun. Lett., vol. 12, no. 8, pp. 545-547, Aug. 2008.

[6] J. Jeganathan, A. Ghrayeb, L. Szczecinski, and A. Ceron, "Space shift keying modulation for MIMO channels", IEEE Trans. Wireless Commun., vol. 8, no. 7, pp. 3692-3703, July 2009.

[7] L. Xiao, L. Greenstein, N. Mandayam, and W. Trappe, "Using the physical layer for wireless authentication in time-variant channels", IEEE Trans. Wireless Commun., vol. 7, no. 7, pp. 2571-2579, July 2008.

[8] A. Alshamali and B. Quza, "Performance of spatial modulation in correlated and uncorrelated Nakagami fading channel", J. Coтmun., vol. 4, no. 3, pp. 170-174, Apr. 2009.

[9] J. Jeganathan, A. Ghrayeb, and L. Szczecinski, "Generalized space shift keying modulation for MIMO channels", IEEE Int. Symp. Personal, Indoor, Mobile Radio Commun., pp. 1-5, Sept. 2008.

[10] M. Di Renzo and H. Haas, "A general framework for performance analysis of space shift keying (SSK) modulation for MISO correlated Nakagami-m fading channels", IEEE Trans. Commun., vol. 58, no. 9, Sept. 2010.

[11] M. Di Renzo and H. Haas, "Space shift keying (SSK) modulation with partial channel state information: Optimal detector and performance analysis over fading channels", IEEE Trans. Commun., vol. 58, no. 10, Oct. 2010.

[12] N. Serafimovski, M. Di Renzo, S. Sinanovic, R. Y. Mesleh, and H. Haas, "Fractional bit encoded spatial modulation (FBE-SM)", IEEE Commun. Lett., vol. 14, no. 5, pp. 429-431, May 2010.

[13] M. Di Renzo and H. Haas, "Improving the performance of space shift keying (SSK) modulation via opportunistic power allocation", IEEE Commun. Lett., vol. 14, no. 6, pp. 500-502, June 2010.

[14] R. Y. Mesleh, M. Di Renzo, H. Haas, and P. M. Grant, "Trellis coded spatial modulation", IEEE Trans. Wireless Commun., vol. 9, no. 7, pp. 2349-2361, July 2010.

[15] M. Di Renzo and H. Haas, "Performance analysis of spatial modulation", ICST/IEEE Int. Conf. Commun. Networking in China, pp. 1-7, Aug. 2010 (invited paper).

[16] M. Di Renzo and H. Haas, "On the performance of SSK modulation over multiple-access Rayleigh fading channels", IEEE Global Commun. Conf., pp. 1-6, Dec. 2010.

[17] P. G. Moschopoulos, "The distribution of the sum of independent gamma random variables", Ann. Inst. Statist. Math. (Part A), vol. 37, pp. 541$544,1985$.

[18] S. M. Alamouti, "A simple transmit diversity technique for wireless communications", IEEE J. Sel. Areas Commun., vol. 16, no. 8, pp. 14511458, Oct. 1998.

[19] V. Tarokh, N. Seshadri, and A. R. Calderbank, "Space-time codes for high data rate wireless communication: Performance criterion and code construction", IEEE Trans. Inform. Theory, vol. 44, no. 2, pp. 744-765, Mar. 1998.

[20] J. Mietzner, R. Schober, L. Lampe, W. H. Gerstacker, and P. A. Höher, "Multiple-antenna techniques for wireless communications - A comprehensive literature survey", IEEE Commun. Surveys Tuts., vol. 11, no. 2, pp. 87-105, 2009. 
[21] M. Abramowitz and I. A. Stegun, Handbook of Mathematical Functions with Formulas, Graphs, and Mathematical Tables, New York, Dover, 9th ed., 1972.

[22] A. P. Prudnikov, Y. A. Brychkov, and O. I. Marichev, Integrals and Series. Vol. 3: More Special Functions, 2003.

[23] M. K. Simon and M.-S. Alouini, Digital Communication over Fading Channels: A Unified Approach to Performance Analysis, John Wiley \& Sons, Inc., 1st ed., 2000.

[24] H. L. Van Trees, Detection, Estimation, and Modulation Theory, Part I: Detection, Estimation, and Linear Modulation Theory, John Wiley \& Sons, Inc. 2001, ISBNs: 0-471-09517-6.

[25] D. Tse and P. Viswanath, Fundamentals of Wireless Communication, Cambridge University Press, 2005.

[26] J. Cheng and T. Berger, "Capacity of a class of fading channels with channel state information (CSI) feedback", Allerton Conference on Communication, Control, and Computing, pp. 1152-1160, Oct. 2001.

[27] G. Golub and J. Welsch, "Calculation of gauss quadrature rules", Math. Comput., vol. 23, pp. 221-230, Apr. 1969.

[28] M.-S. Alouini, A. Abdi, and M. Kaveh, "Sum of gamma variates and performance of wireless communication systems over Nakagami- $m$ fading channels", IEEE Trans. Veh. Technol., vol. 50, no. 6, pp. 14711480, Nov. 2001.

[29] Z. Wang and G. B. Giannakis, "A simple and general parameterization quantifying performance in fading channels", IEEE Trans. Commun., vol. 51, no. 8, pp. 1389-1398, Aug. 2003.

[30] A. Wittneben, "A new bandwidth efficient transmit antenna modulation diversity scheme for linear digital modulation", IEEE Int. Conf. Commun., vol. 3, pp. 1630-1634, May 1993.

[31] J. H. Winters, "The diversity gain of transmit diversity in wireless systems with Rayleigh fading”, IEEE Int. Conf. Commun., vol. 2, pp. 1121-1125, May 1994.

[32] V. Weerackody, "Diversity for direct-sequence spread spectrum system using multiple transmit antennas", IEEE Int. Conf. Commun., vol. 3, pp. 1775-1779, May 1993.

[33] M. Di Renzo, L. A. Annoni, F. Graziosi, and F. Santucci, "A novel class of algorithms for timing acquisition of differential transmitted reference UWB receivers: Architecture, performance analysis and system design", IEEE Trans. Wireless Commun., vol. 7, no. 6, pp. 2368-2387, June 2008.

[34] S. W. Golomb and G. Gong, Signal Design for Good Correlation: For Wireless Communication, Cryptography, and Radar, Cambridge University Press, July 2005.

[35] J. A. Ney da Silva and M. L. R. de Campos, "Spectrally efficient UWB pulse shaping with application in orthogonal PSM", IEEE Trans. Commun., vol. 55, no. 2, pp. 313-322, Feb. 2007.

[36] J. P. Kermoal, L. Schumacher, K. I. Pedersen, P. E. Mogensen, and F. Frederiksen, "A stochastic MIMO radio channel model with experimental validation", IEEE J. Sel. Areas Commun., vol. 20, no. 6, pp. 1211-1226, Aug. 2002.

[37] W. Weichselberger, M. Herdin, H. Özcelik, E. Bonek, "A stochastic MIMO channel model with joint correlation of both link ends", IEEE Trans. Wireless Commun., vol. 5, no. 1, pp. 90-100, Jan. 2006.

[38] M. Chiani, M. Z. Win, and A. Zanella, "On the capacity of spatially correlated MIMO Rayleigh-fading channels", IEEE Trans. Inform. Theory, vol. 49, no. 10 , pp. 2363-2371, Oct. 2003

[39] G. Abreu, C. Mitchell, and R. Kohno, "Jitter-robust orthogonal hermite pulses for ultra wideband impulse radio communications", EURASIP J. Applied Signal Processing, vol. 49, no. 3, pp. 369-381, Mar. 2005.

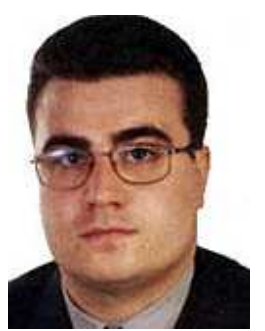

Marco Di Renzo (SM'05-AM'07-M'09) was born in L'Aquila, Italy, in 1978. He received the Laurea (cum laude) and the Ph.D. degrees in Electrical and Information Engineering from the Department of Electrical and Information Engineering, University of L'Aquila, Italy, in April 2003 and January 2007, respectively.

From August 2002 to January 2008, he was with the Center of Excellence for Research DEWS, University of L'Aquila, Italy. From February 2008 to April 2009, he was a Research Associate with the Telecommunications Technological Center of Catalonia (CTTC), Barcelona, Spain. From May 2009 to December 2009, he was a Research Fellow with the Institute for Digital Communications (IDCOM), The University of Edinburgh, Edinburgh, Scotland, United Kingdom (UK).

Since January 2010, he has been a Researcher ("Chargé de Recherche") with the French National Center for Scientific Research (CNRS), and a research staff member of the Laboratory of Signals and Systems (LSS), a joint research laboratory of the CNRS, the École Supérieure d'Électricité (SUPÉLEC), and the University of Paris-Sud XI, Paris, France. His main research interests are in the area of wireless communication theory, signal processing, and information theory.

In December 2004, he was a co-founder of WEST Aquila s.r.l. (Wireless Embedded Systems Technologies L'Aquila), an R\&D Spin-Off of the Department of Electrical and Information Engineering, and the Center of Excellence for Research DEWS, where he currently holds the position of Senior Research Engineer in wireless communications. In 2006, he was a Visiting Scholar with the Mobile and Portable Radio Research Group (MPRG), in the Bradley Department of Electrical and Computer Engineering, Virginia Polytechnic Institute and State University, USA.

Dr. Di Renzo was awarded a special mention for the outstanding fiveyear (1997-2003) academic career from the University of L'Aquila, Italy; a three-year Ph.D. fellowship (ranked 1st) from the Department of Electrical and Information Engineering, University of L'Aquila, Italy, and THALES Communications s.p.a, Land and Joint Systems Division of Advanced Studies, Chieti, Italy; and a personal "Torres Quevedo" Grant (PTQ-08-01-06437) from the "Ministry of Science and Innovation" in Spain for his research on ultra wide band wireless systems and cooperative localization for wireless sensor networks.

Dr. Di Renzo is a Member of the IEEE and IEEE Communications Society, and serves as reviewer for transaction journals and international conferences. He served as Publicity Chair of the 2010 International Conference on Mobile Lightweight Wireless Systems (Mobilight). He also serves as Technical Program Committee (TPC) member and Session Chairman of several international conferences in communications.

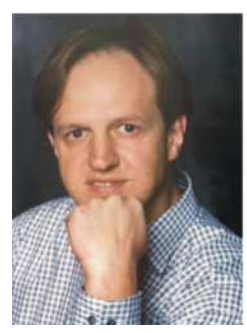

Harald Haas (SM'98-AM'00-M'03) received the $\mathrm{Ph} . \mathrm{D}$. degree from the University of Edinburgh in 2001. His main research interests are in the areas of wireless system design/analysis and digital signal processing, with a particular focus on interference aware MAC protocols, multiuser access, link adaptation, scheduling, dynamic resource allocation, multiple antenna systems and optical wireless communication.

He joined the International University Bremen (Germany), now Jacobs University Bremen, in September 2002 where he is now Honorary Professor of electrical engineering. In June 2007, he joined the University of Edinburgh (Scotland/UK) where he is Professor of Mobile Communications in the Institute for Digital Communications (IDCOM).

Haas received a best paper award at the International Symposium on Personal, Indoor and Mobile Radio Communications (PIMRC) in Osaka/Japan in 1999 and holds more than 15 patents in the area of wireless communications. Haas contributed a chapter to the "Handbook of Information Security" entitled "Air Interface Requirements for Mobile Data Services" by John Wiley \& Sons, Inc. He co-authors a book entitled "Next Generation Mobile Access Technologies: Implementing TDD" with Cambridge University Press. His work on optical wireless communication was selected for publication in "100 Produkte der Zukunft (100 Products of the Future)" authored by Nobel Laureate T. W. Hänsch. Since 2007, Haas is a Regular High Level Visiting Scientist supported by the Chinese " 111 program" at Beijing University of Posts and Telecommunications. 